\title{
Conformally invariant scaling limits in planar critical percolation*
}

\author{
Nike Sun $^{\dagger}$ \\ Department of Statistics, Stanford University \\ 390 Serra Mall \\ Stanford, CA 94305 \\ e-mail: nikesun@stanford.edu
}

\begin{abstract}
This is an introductory account of the emergence of conformal invariance in the scaling limit of planar critical percolation. We give an exposition of Smirnov's theorem (2001) on the conformal invariance of crossing probabilities in site percolation on the triangular lattice. We also give an introductory account of Schramm-Loewner evolutions $\left(\mathrm{SLE}_{\kappa}\right)$, a one-parameter family of conformally invariant random curves discovered by Schramm (2000). The article is organized around the aim of proving the result, due to Smirnov (2001) and to Camia and Newman (2007), that the percolation exploration path converges in the scaling limit to chordal SLE 6 . No prior knowledge is assumed beyond some general complex analysis and probability theory.
\end{abstract}

AMS 2000 subject classifications: Primary 60K35, 30C35; secondary $60 \mathrm{~J} 65$.

Keywords and phrases: Conformally invariant scaling limits, percolation, Schramm-Loewner evolutions, preharmonicity, preholomorphicity, percolation exploration path.

Received August 2011.

\section{Contents}

1 Introduction . . . . . . . . . . . . . . . . . 156

1.1 Critical percolation . . . . . . . . . . . . . . 157

1.2 Conformal invariance . . . . . . . . . . . . . . . . . . . . 159

1.3 The percolation scaling limit $\ldots \ldots \ldots \ldots$

1.3.1 Smirnov's theorem on crossing probabilities . . . . . . . 160

1.3.2 Schramm-Loewner evolutions . . . . . . . . . . . . . 161

1.3.3 Percolation exploration path and convergence to $\mathrm{SLE}_{6} \ldots 163$

1.3.4 Outline of remaining sections . . . . . . . . . . . 163

2 Brownian motion . . . . . . . . . . . . . . . . . . 165

2.1 Conformal invariance of planar Brownian motion . . . . . . . 165

2.2 Harmonic functions and the Dirichlet problem . . . . . . . . . . 168

3 Percolation and Smirnov's theorem on crossing probabilities . . . . . . 170

3.1 Statement of Smirnov's theorem . . . . . . . . . . . . . . 170

* This is an original survey paper.

${ }^{\dagger}$ Partially supported by Department of Defense (AFRL/AFOSR) NDSEG Fellowship. 
3.2 FKG, BK, and RSW inequalities . . . . . . . . . . . 172

3.3 Preharmonic functions . . . . . . . . . . . . . . . . . 173

3.3.1 Cauchy-Riemann equations . . . . . . . . . . . 173

3.3 .2 Integral relations . . . . . . . . . . . . . . 176

3.4 Completing the proof . . . . . . . . . . . . . . . 177

3.5 The percolation exploration path . . . . . . . . . . . 179

4 Schramm-Loewner evolutions . . . . . . . . . . . . . . . . . 179

4.1 Normalizing the conformal maps . . . . . . . . . . . . . . . 179

4.1 .1 Boundary behavior . . . . . . . . . . . . . . . 180

4.1.2 Half-plane capacity . . . . . . . . . . . . . . . . 180

4.2 The Loewner differential equation and SLE . . . . . . . . . . 182

4.3 Characterization of $\mathrm{SLE}_{6} \ldots \ldots \ldots \ldots \ldots$

4.4 Capacity estimates for the LDE . . . . . . . . . . 187

5 Scaling limits . . . . . . . . . . . . . . . . . . . . . 190

5.1 Systems of random curves . . . . . . . . . . . . . 190

5.2 Hölder, tortuosity, and dimension bounds . . . . . . . . . . 192

5.3 Proof of tightness . . . . . . . . . . . . . . . . . . . 194

6 Limit of the exploration path . . . . . . . . . . . . . . . 195

6.1 Admissible domains and discrete approximation . . . . . . . . 196

6.2 Convergence of slit domains . . . . . . . . . . . . . . . . 197

7 Conclusion . . . . . . . . . . . . . . . . . . 200

References . . . . . . . . . . . . . . . . . . . . . . . 201

\section{Introduction}

The field of percolation theory is motivated by a simple physical question: does water flow through a rock? To study this question, Broadbent and Hammersley [28] developed in 1957 the following model for a random porous medium. View the material as an undirected graph $G$, a set of vertices $V$ joined by edges $E$. In the percolation literature the vertices are called sites and the edges bonds. In site percolation, each site is independently chosen to be open or closed with probabilities $p$ and $1-p$ respectively (we refer to $p$ as the "site probability"). The closed sites are regarded as blocked; the open sites, together with the bonds joining them, form the open subgraph, through which water can flow. Percolation theory is the study of the connected components of the open subgraph, called the open clusters. Fig. 1 shows site percolation at $p=1 / 2$ on the triangular lattice $T$ (blue $=$ open, yellow $=$ closed), with the open subgraph highlighted.

Formally, a (site) percolation configuration is a random element $\omega$ of $(\Omega, \mathcal{F})$ where $\Omega=\{0,1\}^{V}(0=$ closed, $1=$ open $)$ and $\mathcal{F}$ is the usual product $\sigma$ algebra generated by the projections $\omega \mapsto \omega_{i}$. Let $\mathbb{P}_{p} \equiv \mathbb{P}_{p}^{\mathrm{s}}$ denote the probability measure on $(\Omega, \mathcal{F})$ induced by percolation with site probability $p$.

This basic model is of course open to any number of variations. An important one is bond percolation, defined similarly except that edges (bonds) rather than vertices are chosen to be open independently with probability $p$, and the 


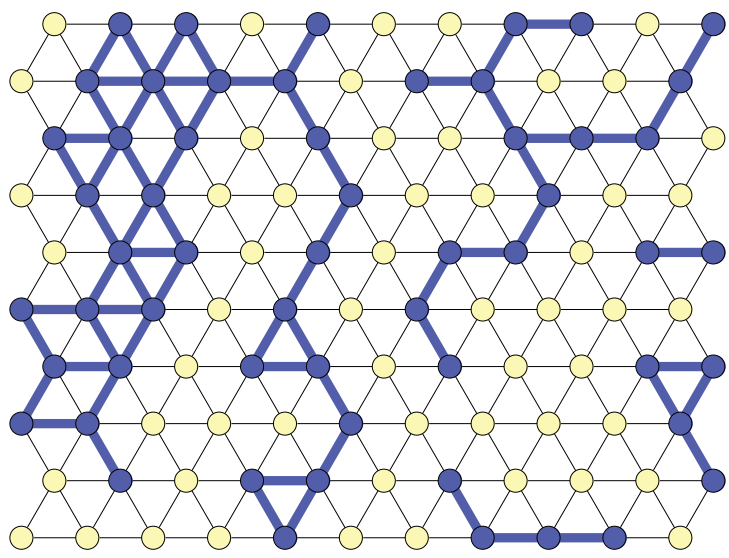

Fig 1: Site percolation on $T$

open subgraph is induced by the open edges. Denote the resulting probability measure on $\{0,1\}^{E}$ by $\mathbb{P}_{p}^{\mathrm{b}}$. In the physical interpretation, in site percolation, water is held mainly in the sites or "pockets" and flows between adjacent pockets - whereas in bond percolation, water is held mainly in the bonds or "channels," and the sites express the adjacency of channels. Historically, bond percolation has been studied more extensively than site percolation; however, bond percolation on a graph $G$ is equivalent to site percolation on the covering graph of $G$.

Percolation is a minimal model which nonetheless captures aspects of the mechanism of interest - that of water seeping through rock - and provides qualitative and quantitative predictions. Since its introduction it has developed into an extremely rich subject, extensively studied by physicists and mathematicians. In this article we focus on one special facet of the theory, and so will certainly miss mentioning many interesting results. The interested reader is referred to the books $[23,47,48,65]$ for accounts of the mathematical theory. For an introduction to the extensive physics literature see [112].

\subsection{Critical percolation}

In percolation, a natural property to consider is the existence of an infinite open cluster: suppose $G=(V, E)$ is countably infinite and connected, and consider site percolation on $G$. For each site $x \in G$ let $C_{x}$ denote the open cluster containing $x$ (with $C_{x}=\varnothing$ if $x$ does not belong to an open cluster). We say that $x$ percolates if $\left|C_{x}\right|=\infty$; the physical interpretation is that $x$ "gets wet." The (site) percolation probability is defined to be

$$
\theta_{x}(p) \equiv \theta_{x}^{\mathrm{s}}(p) \equiv \mathbb{P}_{p}^{\mathrm{s}}\left(\left|C_{x}\right|=\infty\right),
$$

a non-decreasing function of $p$. Because $G$ is connected, for any $x, y \in G, \theta_{x}(p)$ and $\theta_{y}(p)$ must be both positive or both zero. Thus we can define a critical 
(site) probability for the graph $G$ as

$$
p_{c} \equiv p_{c}^{\mathrm{s}}(G) \equiv \inf \left\{p: \theta_{x}(p)>0\right\}
$$

where $x$ is any site in $G$. The analogous quantities for bond percolation are denoted $\theta_{x}^{\mathrm{b}}(p)$ and $p_{c}^{\mathrm{b}}$.

We say percolation occurs in the graph if $\left|C_{x}\right|=\infty$ for some $x \in G$. A common feature of many models in statistical physics is the phase transition, where physical properties of the system undergo abrupt changes as a parameter passes a critical theshold. This is the case in percolation if $G$ has a translation invariance, for example, if $G$ is a lattice, or $G=G_{0} \times \mathbb{Z}$ for some base graph $G_{0}$ : in this setting, the Kolmogorov zero-one law implies that the total number of infinite open clusters is a constant in $\{0,1, \infty\}$. The number is zero below $p_{c}$ and positive above $p_{c}$, so a sharp transition occurs at $p_{c}$. If $G$ is a lattice, $p_{c} \in(0,1)$ (e.g. by $(1.1)$ ), and if an infinite open cluster exists then it is unique ([7], see also $[23,29])$. For an example of a graph $G_{0} \times \mathbb{Z}$ with infinitely many infinite open clusters see [14].

The effort to determine exact values for $p_{c}$ in various models, and to complete the picture of what happens at or near $p_{c}$, has led to a wealth of interesting probabilistic and combinatorial techniques. It was shown by Grimmett and Stacey [49] that if $G=(V, E)$ is countably infinite and connected with maximum vertex degree $\Delta<\infty$, then

$$
\frac{1}{\Delta-1} \leq p_{c}^{\mathrm{b}} \leq p_{c}^{\mathrm{s}} \leq 1-\left(1-p_{c}^{\mathrm{b}}\right)^{\Delta-1}
$$

Kesten proved that $p_{c}^{\mathrm{b}}\left(\mathbb{Z}^{2}\right)=1 / 2$ [64]. He also proved that $p_{c}^{\mathrm{b}}\left(\mathbb{Z}^{d}\right)=(1+$ $o(1)) /(2 d)$ as $d \rightarrow \infty[66]$; better estimates were obtained by Hara and Slade ([50-52], see also [22]). For $\Lambda=T$, Kesten showed $p_{c}=1 / 2$ [65]. There are still famous open problems remaining in this field; for example, it is conjectured that $\theta_{x}^{\mathrm{b}}\left(p_{c}^{\mathrm{b}}\left(\mathbb{Z}^{d}\right)\right)=0$ for all $d \geq 2$, but this has been proved only for $d=2[53,64]$ and $d \geq 19$ [50] (see also [47, Ch. 10-11]). It is also known that $\theta_{x}\left(p_{c}(G)\right)=0$ for both site and bond percolation on on any $G$ which is a Cayley graph of a finitely generated nonamenable group $[15,16]$.

The percolation phenomenon is also defined for a sequence of finite graphs $G_{n}=\left(V_{n}, E_{n}\right)$ with $\left|V_{n}\right| \rightarrow \infty$, where the analogue of the infinite component is the linear-sized or "giant" component. This has been studied most famously in the case of the Erdős-Rényi random graphs $\left(G_{n}\right.$ is the complete graph $\left.K_{n}\right)$, where it was found that around $p_{n}=1 / n$ the size of the largest component has a "double jump" from $O(\log n)$ to $n^{2 / 3}$ to linear; see [21] for historical background and references. Extremely detailed results are known about the structure of the open subgraph near criticality (see e.g. [38] and the references therein). There has also been work to obtain analogous results for other graph sequences: for example for increasing boxes in $\mathbb{Z}^{d}[24]$, for vertex-transitive graphs ([25-27, 89]), and for expander graphs [8]. 


\subsection{Conformal invariance}

The purpose of this article is to explore the limiting structure of the percolation configuration in the scaling limit of increasingly fine lattice approximations to a fixed continuous planar domain $D$ (a nonempty proper open subset of $\mathbb{C}$ which is connected and simply connected). That is, for a planar lattice $\Lambda$, we consider finite subgraphs $G^{\delta} \subset \delta \Lambda$ which "converge" to $D$ as the mesh $\delta$ decreases to zero (to be formally defined later).

It has been predicted by physicists that many classical models (percolation, Ising, FK) at criticality have scaling limits which are conformally invariant and universal. Recall that if $U \subseteq \mathbb{C}$ is an open set, $f: U \rightarrow \mathbb{C}$ is said to be conformal if it is holomorphic (complex-differentiable) and injective. ${ }^{1}$ Conformal maps are so called because they are rigid, in the sense that they behave locally as a rotation-dilation. The Riemann mapping theorem states that for any planar domains $D, D^{\prime}$, there is a conformal bijection $\varphi: D \rightarrow D^{\prime}$. Roughly speaking, conformal invariance means that the limiting (random) behavior of the model on $D^{\prime}$ is the same (in law) as the image under $\varphi$ of the limiting behavior on $D$. Universality means that the limiting behavior is not lattice-dependent.

The physics prediction seems quite surprising because the lattices themselves are certainly not conformally invariant. However, one might expect that lattice percolation on increasingly small scales becomes essentially "locally determined," with the global lattice structure becoming insignificant. Conformal maps behave locally as rotation-dilations and so preserve local structure, so the conformal invariance property means heuristically that the scaling limit of lattice percolation is locally scale-invariant and rotation-invariant. Scale-invariance follows essentially by definition of the scaling limit, and rotation-invariance can be hoped for based on the symmetry and homogeneity of the lattice.

In fact there is a classical example of a conformally invariant scaling limit: the planar Brownian motion, which we discuss in $\S 2$. This is the process $W_{t}=$ $W_{t}^{1}+i W_{t}^{2}$ where the $W_{t}^{i}$ are independent standard Brownian motions in $\mathbb{R}$. By Donsker's invariance principle, the universal scaling limit of planar random walks with finite variance is $\mu t+A W_{t}$ where $\mu \in \mathbb{R}^{2}, A \in \mathbb{R}^{2 \times 2}$ (see e.g. [58]). Using the idea of "local determination" described above, Lévy deduced in the 1930s that planar Brownian motion is conformally invariant, up to a time reparametrization [80]. Lévy's proof, however, was not completely rigorous, and most modern proofs of this result use the theory of stochastic integrals (developed by Itō in the 1950s). Underlying this conformal invariance are some important connections between Brownian motion and harmonic functions, and we will make precise some of these connections which were first observed by Kakutani [57].

\footnotetext{
${ }^{1}$ Some authors more broadly define a conformal map to be any map which is holomorphic with non-vanishing derivative. Basic facts from complex analysis used in this article may be found in e.g. $[1,113]$.
} 


\subsection{The percolation scaling limit}

This article describes two major breakthroughs, due to Schramm [94] and Smirnov $[105,108]$, which gave the rigorous identification in the early 2000s of the scaling limit of critical percolation on the triangular lattice $T$.

\subsubsection{Smirnov's theorem on crossing probabilities}

Langlands, Pouliot, and Saint-Aubin [69], based on experimental observations and after conversations with Aizenman, conjectured that critical lattice percolation has a conformally invariant scaling limit. Some mathematical evidence was provided by Benjamini and Schramm [17], who proved that a different but related model, Voronoi percolation, is invariant with respect to a conformal change of metric.

Using non-rigorous methods of conformal field theory (CFT), physicists were able to give very precise predictions about various quantities of interest in planar critical percolation. Cardy $[33,34]$ notably derived an exact formula for the (hypothetical) limiting probability of an open crossing between disjoint boundary arcs of a planar domain. Carleson made the important observation that this formula has a remarkably simple form when the domain is an equilateral triangle (see §3.4). However, for years mathematicians were unable to rigorously justify the CFT methods used in Cardy's derivation.

In 2001, Smirnov $[105,108]$ proved that for site percolation on the triangular lattice $T$, the limiting crossing probability exists and is conformally invariant, satisfying Cardy's formula. The purpose of $\S 3$ is to give an exposition of this result. The proof is based on the discovery of "preharmonic" functions which encode the crossing probability and converge in the scaling limit to conformal invariants of the domain. ${ }^{2}$ Although the percolation scaling limit is believed to be universal, special symmetries of the triangular lattice play a crucial role in Smirnov's proof, and the result has not been extended to other lattices. For recent work on this question see [12, 19, 20,35].

The exposition of Smirnov's theorem in $\S 3$ is partly based on the one in [23, Ch. 7]. For different perspectives (in addition to the original works of Smirnov) see $[10,48,106]$.

The general principle of preharmonicity and preholomorphicity has been further developed by Chelkak, Hongler, Kemppainen, and Smirnov in establishing conformal invariance in the scaling limit of the critical Ising and FK models $[36,37,54,59,107,109,111]$. Discrete complex analysis appears also in the work of Duminil-Copin and Smirnov [43] determining the connective constant of the hexagonal lattice, which makes substantial progress towards establishing a conformally invariant scaling limit for the self-avoiding walk (SAW). For a more general discussion and references see [44, 110].

\footnotetext{
${ }^{2}$ We use the term preharmonic rather than "discrete harmonic," which also appears in the literature, to avoid confusion with the classical meaning of discrete harmonic (a function whose value at any vertex is the average of the neighboring values) which is not necessarily what is meant here.
} 


\subsubsection{Schramm-Loewner evolutions}

While the notion of a limiting crossing probability is easy to define (though it may not exist), it is not immediately clear how to formally define the "limiting percolation configuration." This notion is discussed in the work of Aizenman [3, 4], and the 1999 work of Aizenman and Burchard [5] shows how to obtain subsequential scaling limits of the percolation configuration. At the time, no direct construction for the limiting object — that is, a construction not involving limits of discrete systems — was available.

Such a construction was discovered in 1999-2000 when, in the course of studying the scaling limit of the loop-erased random walk (LERW), Schramm gave an explicit mathematical description of a one-parameter family of conformally invariant random curves, now called the Schramm-Loewner evolutions (SLE). These curves are characterized by simple axioms which identify them as essentially the universal candidate for the scaling limits of macroscopic interfaces in planar models.

The theory of SLE contains some very beautiful mathematics and is one of the major developments of probability theory within the past decade, and $\S 4$ aims to give an accessible introduction. Here is a brief preview, glossing over all technical details: the SLE are a one-parameter family of self-avoiding ${ }^{3}$ random planar curves $\gamma \equiv \gamma(D ; a, b)$ traveling from $a$ to $b$ in domain $D$, where $a$ is in the boundary $\partial D$ and $b$ is either in $D$ (radial) or elsewhere on $\partial D$ (chordal). The curves satisfy two axioms:

(1) Conformal invariance: if $\varphi$ is a conformal map defined on domain $D$, $\varphi \gamma(D ; a, b)$ has the same law as $\gamma(\varphi D ; \varphi a, \varphi b)$.

(2) Domain Markov property: conditioned on $\gamma[0, t]$, the remaining curve has the same law as $\gamma(D(t), \gamma(t), b)$ where $D(t)$ is the unique connected component of $D \backslash \gamma[0, t]$ whose closure contains $b$ (the "slit domain").

Conformal invariance is expected by physicists as already mentioned, and typically the domain Markov property holds in the discrete setting and is believed to pass to the scaling limit.

Schramm realized that these two properties essentially fully determine the distribution of the curve. His discovery is based on the Loewner differential equation (LDE), which describes the evolution of a self-avoiding curve $\gamma(D ; a, b)$ through the evolution of the corresponding conformal mappings $g_{t}: D(t) \rightarrow D$ (the "slit mappings"). If the domain is the upper-half plane $\mathbb{H}$ with marked boundary points $0, \infty$, we will see that if the maps $g_{t}$ are normalized to "behave like the identity" near $\infty$, then under a suitable time parametrization they satisfy the chordal $L D E$

$$
\dot{g}_{t}(z)=\frac{2}{g_{t}(z)-u_{t}}, \quad g_{0}(z)=z \quad \forall z \in \mathbb{H},
$$

\footnotetext{
${ }^{3} \mathrm{~A}$ formal definition appears in $\S 4.2$. Self-avoiding curves are not necessarily simple; indeed, the scaling limit of the percolation interface will have many double points. Informally, a selfavoiding curve is a curve without transversal self-crossings.
} 


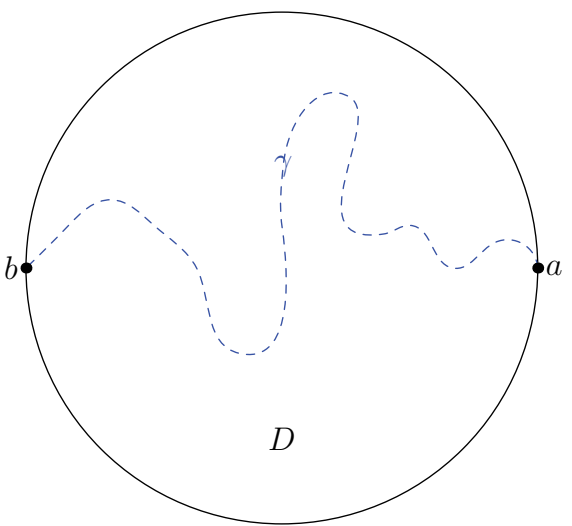

(a) Original SLE curve $\gamma=\gamma_{D, a}$

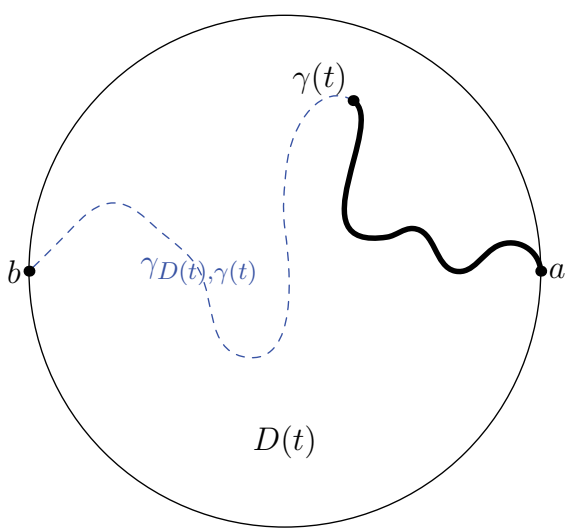

(c) $\gamma_{D(t), \gamma(t)}$ where $D(t)=D \backslash \gamma[0, t]$

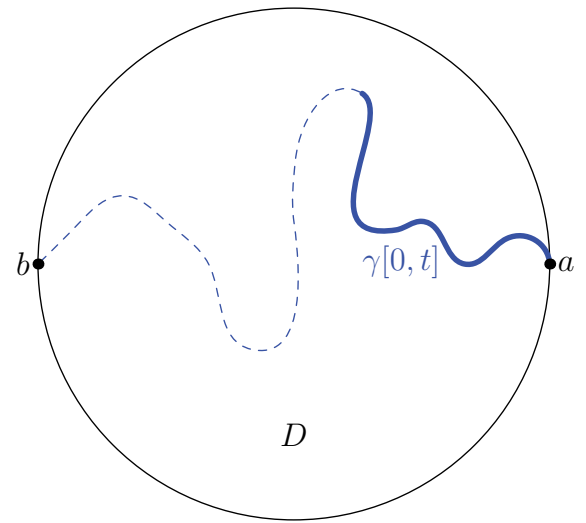

(b) $\gamma[t, \infty)$ conditioned on $\gamma[0, t]$

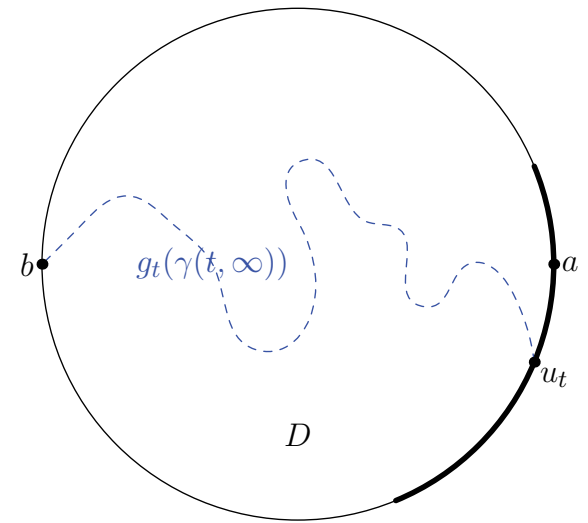

(d) $\gamma_{D(t), \gamma(t)}$ mapped to original domain

Fig 2: Domain Markov property

where $u_{t} \equiv g_{t}(\gamma(t))$ is a continuous real-valued process, called the driving function. (The radial version of the LDE was developed by Charles Loewner in 1923 and used by him to prove a case of the Bieberbach conjecture; see [2].) This equation is remarkable because it encodes the planar curve $\gamma$ in the one-dimensional process $u_{t}$. Given $u_{t}$, one can recover the original curve $\gamma$ by solving the ordinary differential equation above for each $z$ up to time $t$, and setting $\gamma(t)=g_{t}^{-1}\left(u_{t}\right)$.

The SLE axioms imply that if we condition on $\gamma[0, t]$, the image of the remaining curve under $g_{t}$ is distributed as $u_{t}+\tilde{\gamma}$ where $\tilde{\gamma}$ is an independent realization of $\gamma$. Figs. 2a through 2d illustrate this idea. As Schramm noted, this implies that $u_{t}$ must be a Brownian motion, $u_{t}=\mu t+\sqrt{\kappa} W_{t}$. Further, under symmetry assumptions $u$ will have zero drift. The Schramm-Loewner evolutions $\mathrm{SLE}_{\kappa}$ are the curves recovered from the LDE with driving function $u_{t}=\sqrt{\kappa} W_{t}$.

In fact, it will require work to show that " $u_{t}=g_{t}(\gamma(t))$ " is even well-defined, and it is not trivial to find conditions under which a true curve is recovered from 
the LDE. A detailed study of the geometric properties of deterministic Loewner evolutions may be found in [82]. That the $\mathrm{SLE}_{\kappa}$ processes are true curves is a difficult theorem, proved for $\kappa=8$ in [77] and for $\kappa \neq 8$ in [92] (see [72, Ch. 7]). We will not make use of this fact.

In $\S 4$ we follow for the most part Lawler's book [72], which contains far more information than can be covered here. See also the lecture notes $[70,116]$. In the decade since Schramm's original paper there have been many works investigating properties of SLE, e.g. the Hausdorff dimension [9, 11]. Connections to (planar) Brownian motion are discussed in $[71,73,74,76]$. There has been work on characterizing more measures with conformal invariance properties, e.g. the restriction measures of [71]. Sheffield and Werner have given a characterization of conformally invariant loop configurations, the conformal loop ensemble (CLE) [103].

\subsubsection{Percolation exploration path and convergence to $S L E_{6}$}

Schramm conjectured that interfaces in the percolation model converge to forms of $\mathrm{SLE}_{6}$, and the conformal invariance of crossing probabilities was the key to proving this result. In his work [105, 108], Smirnov outlined a proof for the conformal invariance of the full percolation configuration (as a collection of nested curves). His outline was later expanded into detailed proofs in work by Camia and Newman [30, 31].

The proof for the full configuration is beyond the scope of this article, and instead we focus on a single macroscopic interface. The percolation exploration path is defined roughly as follows (the formal definition appears in §3.5): fix two points $a, b$ on the boundary of a simply connected domain. Fix all the hexagons on the counterclockwise arc $\overline{a b}$ to be closed, and all those on $\overline{b a}$ to be open. The exploration path is the interface curve which separates the closed cluster touching $\overline{a b}$ from the open cluster touching $\overline{b a}$. Fig. 3 shows part of an exploration path traveling in $\mathbb{H}$ between 0 and $\infty$. The purpose of $\S 5$ and $\S 6$ is to prove that the exploration path converges to chordal $\mathrm{SLE}_{6}$.

\subsubsection{Outline of remaining sections}

The organization of the remainder of this article is as follows:

- In $\S 2$ we give a proof of the conformal invariance of Brownian motion, and discuss the connection to harmonic functions first developed by Kakutani [57]. The material presented in this section is elementary in nature and can be skipped without loss by readers already familiar with the subject.

- In $\S 3$ we present Smirnov's theorem [105, 108] on the conformal invariance of crossing probabilities in critical percolation on the triangular lattice $T$.

- $\S 4$ is an introduction to the Loewner differential equation and SLE. $\S 4.3$ states a characterization of $\mathrm{SLE}_{6}$ (following Camia and Newman [31]) which will be used in identifying the scaling limit of the exploration path. 


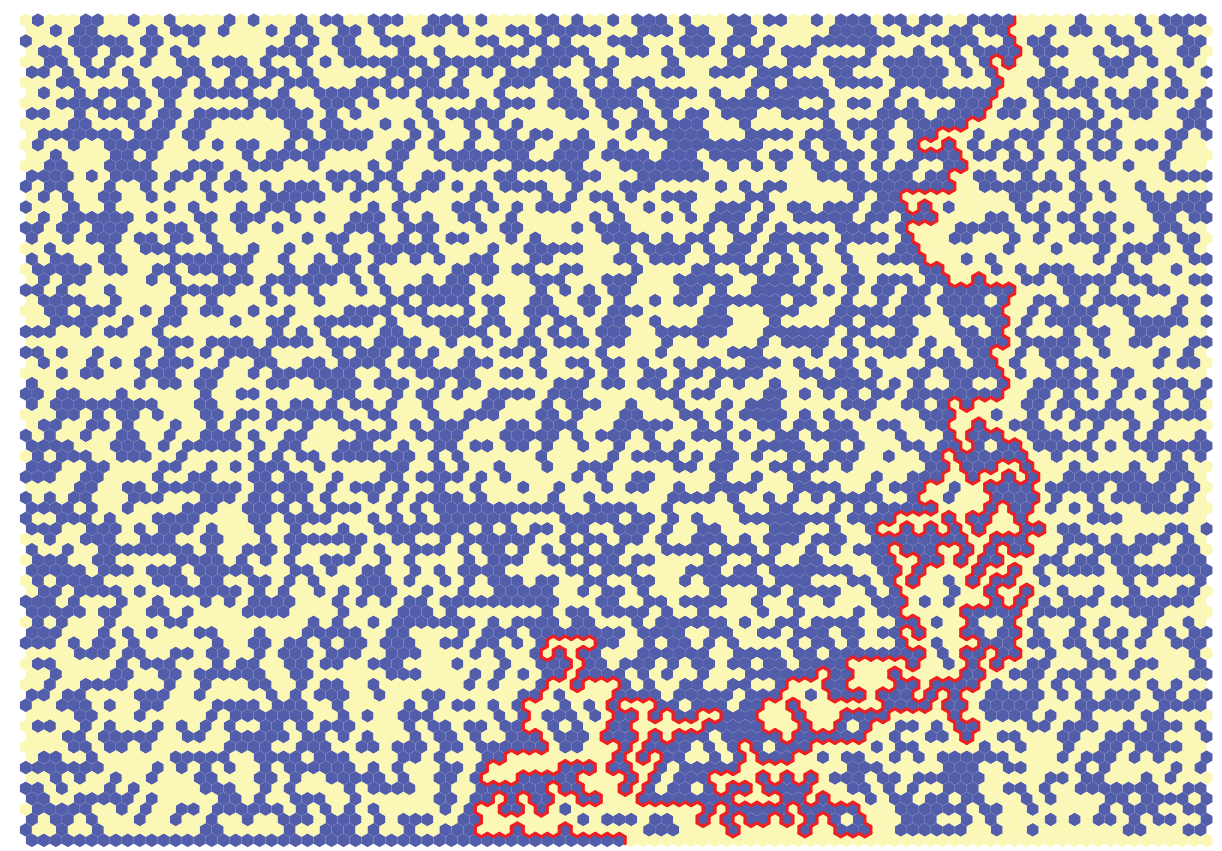

Fig 3: Percolation exploration path

- $\S 5$ presents a result of Aizenman and Burchard [5] which uses a priori estimates (in our case, percolation crossing exponents) to deduce the existence of subsequential weak limits for the percolation exploration path.

- In $\S 6$ we show that all subsequential limits of the exploration path are chordal SLE 6 . Our exposition follows the work of Binder, Chayes and Lei $[19,20]$ and of Camia and Newman [31].

- $\$ 7$ concludes with some additional references and open problems concerning other planar models.

For more accounts on conformal invariance in percolation and convergence to $\mathrm{SLE}_{6}$, see the lecture notes of Werner [117] and of Beffara and Duminil-Copin [13]. For a broad overview of conformally invariant scaling limits of planar models see the survey of Schramm [96].

\section{Acknowledgements}

This survey was originally prepared as an undergraduate paper in mathematics at Harvard University under the guidance of Yum-Tong Siu and Wilfried Schmid, and I thank them both for teaching me about complex analysis, and for their generosity with their time and advice.

I am very grateful to Scott Sheffield for teaching me about percolation and SLE, for answering countless questions and suggesting useful references. I would like to thank Scott Sheffield and Geoffrey Grimmett for carefully reading drafts 
of this survey and making valuable suggestions for improvement which I hope are reflected here.

I thank Amir Dembo, Jian Ding, Asaf Nachmias, David Wilson, and an anonymous referee for many helpful comments and corrections on recent drafts of this survey. I thank Michael Aizenman, Almut Burchard, Federico Camia, Curtis McMullen, Charles Newman, and Horng-Tzer Yau for helpful communications during the course of writing.

\section{Brownian motion}

Some notations: let $B_{r}(z)$ denote the open ball of radius $r$ centered at $z \in \mathbb{R}^{d}$, and $C_{r}(z) \equiv \partial B_{r}(z)$. Let $B_{r} \equiv B_{r}(0), C_{r} \equiv \partial B_{r}$ denote the versions of these which are centered at the origin.

If $(\Omega, \mathcal{F}, \mathbb{P})$ is a probability triple and $\left(\mathcal{F}_{t}\right)_{t \geq 0}$ is an increasing filtration in $\mathcal{F}$, we call $\left(\Omega, \mathcal{F},\left(\mathcal{F}_{t}\right)_{t \geq 0}, \mathbb{P}\right)$ a filtered probability space. For $X \in \mathcal{F}_{0}$, an $\left(\mathcal{F}_{t}\right)$ Brownian motion started at $X$ is a stochastic process $\left(W_{t}\right)_{t \geq 0}$ defined on $(\Omega, \mathcal{F}, \mathbb{P})$ which is $\left(\mathcal{F}_{t}\right)$-adapted and satisfies

1. $W_{0}=X$ a.s.;

2. $W$ is a.s. continuous;

3. for $s<t, W_{t}-W_{s} \sim \mathcal{N}(0, t-s)$ and is independent of $\mathcal{F}_{t}$.

A Brownian motion in $\mathbb{R}^{d}$ is simply a $d$-dimensional vector of independent Brownian motions $\left(W_{t}^{1}, \ldots, W_{t}^{d}\right)$. For $d=2$ we always identify $\mathbb{R}^{2} \cong \mathbb{C}$ and write $W_{t} \equiv W_{t}^{1}+i W_{t}^{2}(t \geq 0)$, referred to as planar or complex Brownian motion. An approximation of a sample path is shown in Fig. 4 (the path becomes lighter over time). For $z \in \mathbb{C}$ let $\mathbb{P}_{z}$ denote the probability measure for Brownian motion started at $z$, and write $\mathbb{E}_{z}$ for expectation with respect to $\mathbb{P}_{z}$. For more on Brownian motion see e.g. [58, 87].

\subsection{Conformal invariance of planar Brownian motion}

The result below tells us that the holomorphic image of a Brownian motion is again a Brownian motion up to a (random) time change. For $D$ a complex domain $^{4}$ and $W$ a Brownian motion started at $z_{0} \in D$, let

$$
\tau_{D} \equiv \inf \left\{t \geq 0: W_{t} \notin D\right\}
$$

denote the exit time of $D$.

Theorem 2.1. Let $\varphi$ be a holomorphic map defined on the complex domain D, and for $W$ a Brownian motion started at $z_{0} \in D$ let $Y_{t}=\varphi\left(W_{t}\right)\left(t<\tau_{D}\right)$. If

$$
Y_{t} \equiv \widetilde{W}_{\sigma(t)}, \quad \sigma(t) \equiv \int_{0}^{t}\left|f^{\prime}\left(W_{s}\right)\right|^{2} d s
$$

then $\widetilde{W}_{t}$ is a Brownian motion started at $\varphi\left(z_{0}\right)$ run up to $\tau_{\varphi(D)}$.

\footnotetext{
${ }^{4}$ Recall that by a complex domain we always mean a nonempty proper open subset of $\mathbb{C}$ which is connected and simply connected.
} 


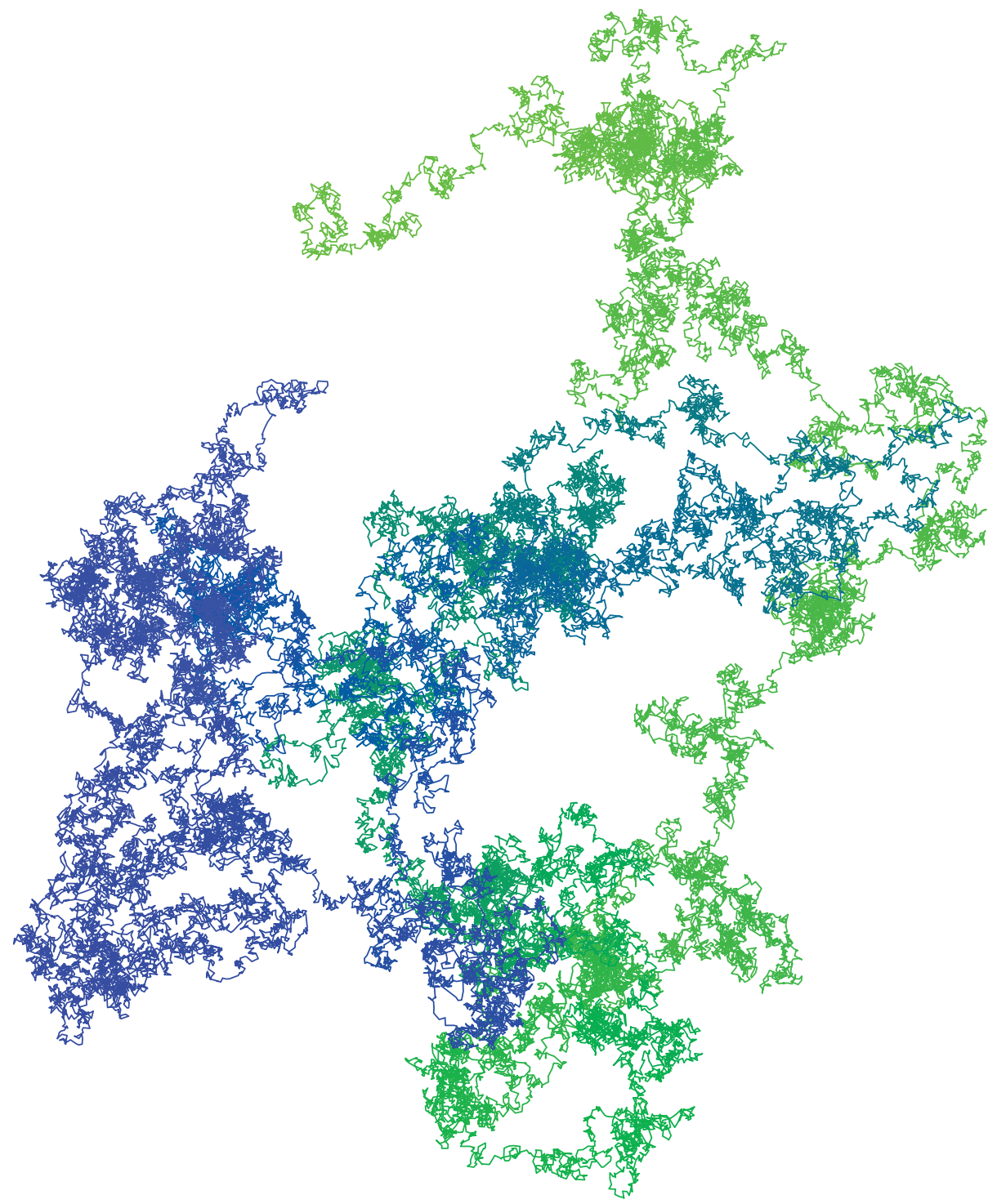

Fig 4: Approximate sample path of Brownian motion

Here is Lévy's heuristic argument [80]: translations and rotations of $\mathbb{C}$ clearly map Brownian motions to Brownian motions, and we have the Brownian scaling $\widetilde{W}_{t}=c W_{t / c^{2}}(c>0)$ for $\widetilde{W}$ another Brownian motion of the same speed as $W$. The stochastic process is determined by its behavior "locally" at each point in time, and near time $t$ we have

$$
\varphi\left(W_{t+h}\right)-\varphi\left(W_{t}\right) \approx \lambda_{t}\left(W_{t+h}-W_{t}\right), \quad \lambda_{t} \equiv \varphi^{\prime}\left(W_{t}\right) .
$$


By rotation-invariance and the Brownian scaling, $\lambda_{t}\left(W_{t+h}-W_{t}\right)=\widetilde{W}_{\left|\lambda_{t}\right|^{2} h}$, so $\varphi(W)$ at time $t$ behaves like a standard Brownian motion "sped up" by a factor of $\left|\lambda_{t}\right|^{2}$.

Below we present the modern proof of this result, which uses stochastic calculus. ${ }^{5}$ In preparation we first recall Itō's formula, Lévy's characterization of Brownian motion, and the Dubins-Schwarz theorem (proofs may be found in e.g. [58]). For two continuous semimartingales $X, X^{\prime}$ we use the notation $\left[X, X^{\prime}\right]$ for their covariation process, and we write $[X] \equiv[X, X]$ for the quadratic variation process of $X$.

Theorem 2.2 (Itō's formula). Let $X$ be a continuous semimartingale: $X_{t}=$ $X_{0}+M_{t}+A_{t}$ where $M$ is a local martingale and $A$ is a finite-variation process. Then $f\left(X_{t}\right)$ is again a continuous semimartingale with

$$
f\left(X_{t}\right)-f\left(X_{0}\right)=\sum_{j=1}^{d} \int_{0}^{t} \partial_{j} f\left(X_{s}\right) d X_{s}^{j}+\frac{1}{2} \sum_{j, k=1}^{d} \int_{0}^{t} \partial_{j} \partial_{k} f\left(X_{s}\right) d\left[X^{j}, X^{k}\right]_{s} .
$$

In differential notation

$$
d f\left(X_{t}\right)=\sum_{j=1}^{d} \partial_{j} f\left(X_{s}\right) d X_{s}^{j}+\frac{1}{2} \sum_{j, k=1}^{d} \partial_{j} \partial_{k} f\left(X_{s}\right) d\left[X^{j}, X^{k}\right]_{s} .
$$

Theorem 2.3 (Lévy's characterization of Brownian motion). Let $\left(X_{t}\right)_{t \geq 0}$ be a continuous adapted process in $\mathbb{R}^{d}$ defined on the filtered probability space $\left(\Omega, \mathcal{F},\left(\mathcal{F}_{t}\right)_{t \geq 0}, \mathbb{P}\right)$ such that

(i) $X_{t}^{j}-X_{0}^{j}$ is a local $\left(\mathcal{F}_{t}\right)$-martingale for $1 \leq j \leq d$;

(ii) the pairwise quadratic variations are $\left[X^{j}, X^{k}\right]_{t}=\delta_{k j}$ t for $1 \leq k, j \leq d$.

Then $X$ is an $\left(\mathcal{F}_{t}\right)$-Brownian motion in $\mathbb{R}^{d}$.

Theorem 2.4 (Dubins-Schwarz theorem). Let $M$ be a continuous local martingale defined on the filtered probability space $\left(\Omega, \mathcal{F},\left(\mathcal{F}_{t}\right)_{t \geq 0}, \mathbb{P}\right)$ with $[M]_{t} \rightarrow \infty$ a.s. If

$$
\tau(s) \equiv \inf \left\{t \geq 0:[M]_{t}>s\right\}, \quad \mathcal{G}_{s} \equiv \mathcal{F}_{\tau(s)},
$$

then $W_{s} \equiv M_{\tau(s)}$ is a $\left(\mathcal{G}_{t}\right)$-Brownian motion, and $M_{t}=W_{[M]_{t}}$.

Proof of Thm. 2.1. Let $u=\operatorname{Re} \varphi$ and $v=\operatorname{Im} \varphi$; we regard $u, v$ as functions on $\mathbb{R}^{2}$ and write $\varphi(x+i y)=u(x, y)+i v(x, y)$. Writing $W_{t}=W_{t}^{1}+i W_{t}^{2}$ and applying Itō's formula (using the indepence of the two components) gives

$$
\begin{aligned}
& d u\left(W_{t}\right)=\partial_{x} u\left(W_{t}\right) d W_{t}^{1}+\partial_{y} u\left(W_{t}\right) d W_{t}^{2}+\frac{1}{2} \Delta u\left(W_{t}\right) d t \\
& d v\left(W_{t}\right)=\partial_{x} v\left(W_{t}\right) d W_{t}^{1}+\partial_{y} v\left(W_{t}\right) d W_{t}^{2}+\frac{1}{2} \Delta u\left(W_{t}\right) d t .
\end{aligned}
$$

\footnotetext{
${ }^{5}$ Stochastic calculus is not heavily used in this article, so the reader not familiar with the subject will not lose much by simply skipping over the places where it appears.
} 
By the Cauchy-Riemann equations,

$$
d[u(W)]_{t}=\left(\left[\partial_{x} u\left(W_{t}\right)\right]^{2}+\left[\partial_{y} u\left(W_{t}\right)\right]^{2}\right) d t=\left|f^{\prime}\left(W_{t}\right)\right|^{2} d t=d[v(W)]_{t}
$$

and

$$
d[u(W), v(W)]_{t}=\left[\partial_{x} u\left(W_{t}\right) \partial_{x} v\left(W_{t}\right)+\partial_{y} u\left(W_{t}\right) \partial_{y} v\left(W_{t}\right)\right] d t=0 .
$$

Further $u, v$ are harmonic (again by the Cauchy-Riemann equations) so $u\left(W_{t}\right)$ and $v\left(W_{t}\right)$ are local martingales, hence time-changed Brownian motions by the Dubins-Schwarz theorem:

$$
u\left(W_{t}\right)=\widetilde{W}_{\sigma(t)}^{1}, \quad u\left(W_{t}\right)=\widetilde{W}_{\sigma(t)}^{2}
$$

for Brownian motions $\widetilde{W^{1}}, \widetilde{W}^{2}$. (One needs to verify that $\sigma(t) \rightarrow \infty$; this follows from holomorphicity of $\varphi$ and the neighborhood recurrence of planar Brownian motion.) Finally, we leave the reader to check that $\left[\widetilde{W}^{1}, \widetilde{W}^{2}\right]=0$; the result then follows from Lévy's characterization of Brownian motion.

\subsection{Harmonic functions and the Dirichlet problem}

Given a domain $D$ and a bounded continuous function $f: \partial D \rightarrow \mathbb{R}$, we say that $u \in C^{2}(D) \cap C(\bar{D})$ solves the Dirichlet problem on $D$ with boundary data $f$ if $u$ is harmonic $(\Delta u=0)$ in $D$ and agrees with $f$ on $\partial D$. We now demonstrate the connection to Brownian motion as first noted by Kakutani [57]. Recall that $\tau_{D}$ denotes the exit time of a Brownian motion from $D$, and let $\tau_{D}^{+} \equiv \inf \left\{t>0: W_{t} \notin D\right\}$. A domain $D$ is said to be regular if for all $z \in \partial D$, $\tau_{D}^{+}=0, \mathbb{P}_{z}$-a.s.

Proposition 2.5. Let $D$ be a bounded regular domain, and let $f: \partial D \rightarrow \mathbb{R}$ continuous. Define $u: \bar{D} \rightarrow \mathbb{R}$ by

$$
u(z)=\left\{\begin{array}{cl}
f(z) & \text { if } z \in \partial D . \\
\mathbb{E}_{z}\left[f\left(W_{\tau_{D}}\right)\right] & \text { if } z \in D .
\end{array}\right.
$$

Then $u$ is a bounded solution to the Dirichlet problem on D with boundary data $f$, and it is the unique such solution.

Proof (sketch). Since $f$ is bounded, $u$ is certainly bounded. Uniqueness is easy: let $v$ be another such solution. Then $v\left(W_{t}\right)$ is a bounded local martingale, hence a uniformly integrable martingale, so by the optional stopping theorem

$$
v(z)=v\left(W_{0}\right)=\mathbb{E}_{z}\left[v\left(W_{\tau_{D}}\right)\right]=\mathbb{E}_{z}\left[f\left(W_{\tau_{D}}\right)\right]=u(z) .
$$

To show $u$ is harmonic we check the local mean-value property, that for each $z \in D$ there exists $r_{0}>0$ such that

$$
u(z)=\frac{1}{2 \pi} \int_{0}^{2 \pi} u\left(z+r e^{i \theta}\right) d \theta
$$


for $0<r \leq r_{0}$ (see e.g. [81]). Let $W_{t}$ be a Brownian motion started at $z$, and let $\sigma=\tau_{B_{r}(z)}$. By the rotational symmetry of Brownian motion, $W_{\sigma}$ is uniformly distributed on $\partial B_{r}(z)$, so the right-hand side of the above is

$$
\begin{aligned}
\mathbb{E}_{z}\left[u\left(W_{\sigma}\right)\right] & =\mathbb{E}_{z}\left[\mathbb{E}_{W_{\sigma}}\left[f\left(W_{\tau_{D}}\right)\right]\right] \quad \text { by definition of } u \\
& =\mathbb{E}_{z}\left[\mathbb{E}_{z}\left[f\left(W_{\tau_{D}}\right) \mid \mathcal{F}_{\sigma}\right]\right] \quad \text { by strong Markov property } \\
& =\mathbb{E}_{z}\left[f\left(W_{\tau_{D}}\right)\right] \quad \text { by iterated expectations } \\
& =u(z) .
\end{aligned}
$$

This shows that $u$ is harmonic. Continuity of $u$ on $\bar{D}$ requires the regularity assumption and we omit the proof here.

Remark 2.6. For some simple domains $U$ a Poisson integral formula gives an explicit mapping $P_{U}$ from a (bounded continuous) function $f$ defined on $\partial U$ to the solution of the Dirichlet problem on $U$ with boundary values $f$. The formulas for the disc and the upper half-plane are well known and will be used in deriving the Loewner differential equation, so we recall them here:

$$
\begin{aligned}
& \left(P_{\mathbb{D}} f\right)\left(r e^{i \theta}\right)=\frac{1}{2 \pi} \int_{0}^{2 \pi} P_{r}(\theta-\varphi) f\left(e^{i \varphi}\right) d \varphi, \quad P_{r}(\theta)=\operatorname{Re}\left(\frac{1+r e^{i \theta}}{1-r e^{i \theta}}\right), \\
& \left(P_{\mathbb{H}} f\right)(x+i y)=\frac{1}{\pi} \int_{\mathbb{R}} Q_{y}(t-x) f(t) d t, \quad Q_{y}(x)=\frac{y}{x^{2}+y^{2}} .
\end{aligned}
$$

For proofs see e.g. $[113,114]$.

Propn. 2.5 also gives us a weaker version of Thm. 2.1, namely, that the hitting distribution of Brownian motion is conformally invariant. Indeed, let $D, D^{\prime}$ be regular domains (say with smooth boundaries), and let $z$ be a point in $D$ and $A$ an $\operatorname{arc}$ on $\partial D$. Let $z^{\prime}=\varphi(z), A^{\prime}=\varphi(A)$, and $W_{t}^{\prime}=\varphi\left(W_{t}\right)$. Then $u(z) \equiv \mathbb{P}_{z}\left(W_{\tau_{D}} \in A\right)$ solves the Dirichlet problem on $D$ with boundary conditions $f=\mathbf{1}_{A}$, while $u^{\prime}(z) \equiv \mathbb{P}_{z^{\prime}}\left(W_{\tau_{D^{\prime}}} \in A^{\prime}\right)$ solves the Dirichlet problem on $D^{\prime}$ with boundary conditions $f^{\prime}=\mathbf{1}_{A^{\prime}}{ }^{6}$ But

$$
u \circ \varphi^{-1}
$$

is also a solution, so by uniqueness we have $u=u^{\prime} \circ \varphi$. Since $A$ was arbitrary the conformal invariance of the hitting distribution follows.

In fact, here is a way to deduce the full conformal invariance of the Brownian path (modulo time reparametrization) from this seemingly weaker result. For each $\epsilon>0$, we approximate the Brownian path by the piecewise linear curve $W_{t}^{(\epsilon)}$ joining the points $z=z_{1}, z_{2}, \ldots$, where $z_{k+1}$ is the first point where the Brownian motion starting from $z_{k}$ hits $B_{\epsilon}\left(z_{k}\right)$. To be precise, we will define a sequence of stopping times $0=\tau_{0}, \tau_{1}, \ldots$ by recursively setting $z_{k}=W_{\tau_{k}}$ and $\tau_{k+1}=\tau_{B_{\epsilon}\left(z_{k}\right)}$. We then make $W_{t}^{(\epsilon)}$ into a continuous stochastic process

\footnotetext{
${ }^{6}$ The indicator functions are discontinuous, but since we assumed $\partial D$ to be smooth we may easily approximate $\mathbf{1}_{A}$ by continuous functions.
} 
by linear interpolation. It is clear that as $\epsilon \rightarrow 0, W_{t}^{(\epsilon)}$ converges a.s. to $W_{t}$ in the topology of uniform convergence. Now, if $\varphi$ is a conformal map, then by the above $\varphi\left(W_{\tau_{k+1}}^{(\epsilon)}\right)$ has the same distribution as the point where a Brownian motion started at $\varphi\left(z_{k}\right)$ first exits the conformal ball $\varphi\left(B_{\epsilon}\left(z_{k}\right)\right)$. It follows that

$\varphi\left(W_{t}^{(\epsilon)}\right)$ converges a.s. as $\epsilon \rightarrow 0$ to a process which is a time reparametrization of Brownian motion.

The proof of Smirnov's theorem for crossing probabilities, in the next section, is to some extent motivated by these simple observations. In particular, conformal invariance of the crossing probabilities will follow naturally from expressing the probabilities in terms of a harmonic function solving some form of Dirichlet problem. The idea of constructing polygonal approximations to a random path will also reappear, in $\S 6$, when we use a modification of this construction to show that the scaling limit of percolation agrees with chordal $\mathrm{SLE}_{6}$.

\section{Percolation and Smirnov's theorem on crossing probabilities}

In this section we present Smirnov's celebrated theorem on the conformal invariance of crossing probabilities in critical percolation on the triangular lattice $T$ [105, 108]. Smirnov's key insight was the discovery of a "preharmonic" function whose evaluation at a certain point gives the crossing probability. As $\delta \rightarrow 0$, these functions converge to a true harmonic function solving a Dirichlet-type problem on $D$, and the theorem follows because the solution to the Dirichlet problem is a conformal invariant.

The first section below gives a formal statement of Smirnov's crossing probabilities theorem.

\subsection{Statement of Smirnov's theorem}

Let $\Lambda=(V, E)$ be a planar lattice. Write $u \sim v$ if $(u, v) \in E$. Site percolation on $\Lambda$ may be visualized as face percolation on the dual lattice $\Lambda^{*}$. We will use blue and yellow in the place of open and closed respectively, particularly to avoid confusion with the topological meanings of those words.

Smirnov's theorem is for site percolation on $T$, shown with its dual hexagonal lattice $H$ in Fig. 5. Each vertex $v \in T$ corresponds to a hexagon $H_{v}$. A triangular vertex means a vertex of $T$, and a triangular path means the polygonal interpolation of a path in $T$, regarded as a polygonal curve in $\mathbb{C}$. Define similarly hexagonal vertex and hexagonal path.

Recall that a domain is a nonempty proper open subset $D \subset \mathbb{C}$ which is simply connected. $D$ is called a Jordan domain if its boundary $\partial D$ is a Jordan curve. In this case if $a, b \in \partial D$ then $\overline{a b}$ denotes the counterclockwise arc from $a$ to $b$ on $\partial D$.

Definition 3.1. A $k$-marked (Jordan) domain is a Jordan domain $D$ together with $k$ distinct points $P_{1}, \ldots, P_{k} \in \partial D$ in counterclockwise order, denoted $D_{k}=\left(D ; P_{1}, \ldots, P_{k}\right)$. Write $A_{j} \equiv A_{j}\left(D_{k}\right)$ for the arc $\overline{P_{j} P_{j+1}}$ (indices taken modulo $k$ ). 


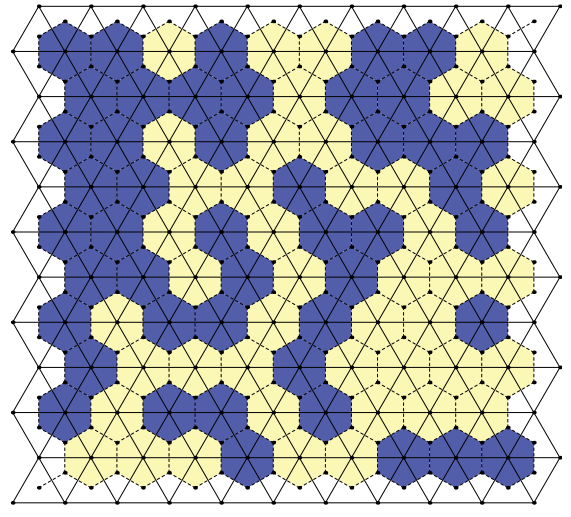

(a) $T$ with dual $H$

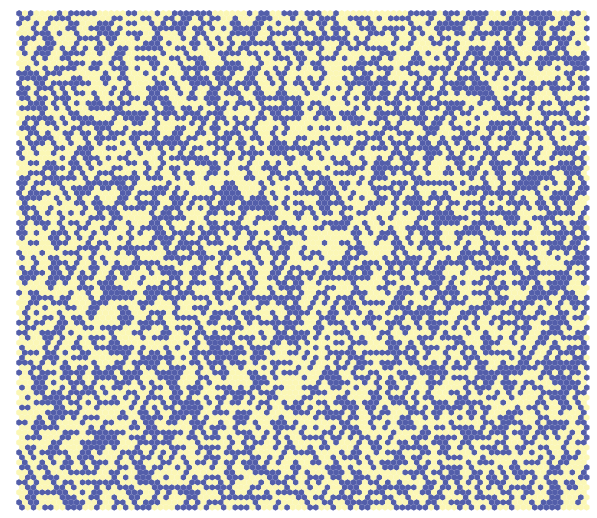

(b) Face percolation on $H$

Fig 5: Site percolation on $T$ as face percolation on $H$

A conformal equivalence of $k$-marked domains $D_{k}, D_{k}^{\prime}$ is a conformal map $\varphi: D \rightarrow D^{\prime}$ with $\varphi: P_{j} \mapsto P_{j}^{\prime}$ for all $j$.

Definition 3.2. A $k$-marked discrete domain with mesh $\delta$ is a $k$-marked domain $D_{k}^{\delta}=\left(D^{\delta} ; P_{1}^{\delta}, \ldots, P_{k}^{\delta}\right)$ such that $D^{\delta}$ is the interior of a union of closed hexagonal faces of $\delta H$, and each $P_{j}^{\delta}$ is a vertex of $\delta H$ incident to a unique hexagon inside $D$.

Let $\mathbb{P}_{p}^{\delta}$ denote the law of percolation at probability $p$ on $D_{4}^{\delta}$ (more precisely, face percolation on the hexagons inside $D^{\delta}$ ). We say a blue (yellow) crossing of $D_{4}^{\delta}$ occurs if there is a path of blue (yellow) hexagons joining $A_{1}\left(D_{4}^{\delta}\right)$ to $A_{3}\left(D_{4}^{\delta}\right)$. Let $A_{j}^{+}$denote the set of hexagons in the external boundary of $D^{\delta}$ with at least one edge contained in $A_{j}$; it is sometimes helpful to think of the $A_{j}^{+}$as having predetermined colors, and these are shown with darker shading in the figures.

We now clarify the sense in which the $D_{4}^{\delta}$ approximate $D_{4}$ as $\delta \downarrow 0$. To do so we need a formal definition of "curves modulo reparametrization":

Definition 3.3. A distance function on the space $\mathcal{C}_{0}$ of continuous functions $f:[0,1] \rightarrow \mathbb{R}^{d}$ is given by

$$
d_{\mathcal{U}}\left(f_{1}, f_{2}\right) \equiv \inf _{\phi}\left\|f_{1} \circ \phi-f_{2}\right\|_{\infty}
$$

where the infimum is taken over increasing homeomorphisms $\phi$ of $[0,1]$. Say that $f_{1}, f_{2}$ are equivalent up to reparametrization, denoted $f_{1} \sim f_{2}$, if $d_{\mathcal{U}}\left(f_{1}, f_{2}\right)=0$. A curve is an element of the space $\mathcal{C} \equiv \mathcal{C}_{0} / \sim$, and we refer to the metric $d_{\mathcal{U}}$ on $\mathcal{C}$ as the uniform metric.

The space $\mathcal{C}$ is separable and complete. For $\gamma \in \mathcal{C}$ we will frequently abuse notation and write $\gamma$ also for a representative of $\gamma$ in $\mathcal{C}_{0}$.

Definition 3.4. For bounded $k$-marked domains $D_{k}^{\delta}, D_{k}$, say that $D_{k}^{\delta}$ converges uniformly to $D_{k}$ if $A_{j}\left(D_{k}^{\delta}\right) \rightarrow A_{j}\left(D_{k}\right)$ uniformly for all $j$. 
Recall that the critical probability for site percolation on $T$ is $p_{c}=1 / 2$; from now on we write $\mathbb{P}^{\delta} \equiv \mathbb{P}_{1 / 2}^{\delta}$. Here then is a statement of Smirnov's theorem confirming the Langlands et al. conjecture:

Theorem 3.5 (Smirnov's theorem on crossing probabilities [105, 108]). Let $D_{4}^{\delta}$ be four-marked discrete domains converging uniformly to the bounded fourmarked Jordan domain $D_{4}$. Then

$$
\Phi^{\delta}\left(D_{4}^{\delta}\right) \equiv \mathbb{P}^{\delta}\left(D_{4}^{\delta} \text { has a blue crossing }\right)
$$

converges to a limit $\Phi\left(D_{4}\right) \in(0,1)$ which is conformally invariant.

Remark 3.6. It will be clear from the proof that the above assumption of uniform convergence is unnecessarily strong. In fact, in order to prove the scaling limit for the exploration path we will require a version of Thm. 3.5 which holds for a more general notion of discrete approximation, which will be conceptually straightforward but slightly tricky to describe. With a view towards keeping the exposition simple, we ignore the issue for now and address it in $\S 6.1$.

\subsection{FKG, BK, and $R S W$ inequalities}

In this section are collected some results of basic percolation theory which will be needed in the proof.

For the first two results, the FKG and BK inequalities, the graph structure is irrelevant so we return to a more general setting: let $\Omega \equiv\{0,1\}^{V}$, and let $\mathbb{P}_{p}$ denote the law of site percolation at probability $p$ on $V$. For $\omega, \omega^{\prime} \in \Omega$, say $\omega \leq \omega^{\prime}$ if the inequality holds coordinate-wise. A random variable $X$ on $(\Omega, \mathcal{F})$ is called increasing if $X(\omega) \leq X\left(\omega^{\prime}\right)$ for all $\omega \leq \omega^{\prime}$, and an event $A \in \mathcal{F}$ is increasing if $\mathbf{1}_{A}$ is increasing. The following inequality tells us that increasing events are, as naturally expected, positively correlated:

FKG inequality (Harris [53], Fortuin, Kasteleyn, Ginibre [46]). If $X, Y$ are increasing random variables on $(\Omega, \mathcal{F})$, then $\mathbb{E}_{p}(X Y) \geq\left(\mathbb{E}_{p} X\right)\left(\mathbb{E}_{p} Y\right)$. In particular, increasing events are positively correlated.

Here is another useful result which gives bounds in the other direction: for $A, B \in \mathcal{F}$ increasing events depending only on the states of finitely many sites, let $A \square B$ denote the event that there are disjoint witnesses for $A$ and $B$ i.e., $\omega \in A \square B$ if and only if there exist disjoint sets $I_{A}, I_{B} \subset V$ such that $\left.\omega^{\prime}\right|_{I_{A}}=\left.\omega\right|_{I_{A}}$ implies $\omega^{\prime} \in A$, and $\left.\omega^{\prime}\right|_{I_{B}}=\left.\omega\right|_{I_{B}}$ implies $\omega^{\prime} \in B$. The following inequality says that the existence of disjoint witnesses for two events is less likely than the simple intersection of the two events:

BK inequality (van den Berg, Kesten [115]). If $A, B \in \mathcal{F}$ are both increasing events depending only on the states of finitely many edges, then $\mathbb{P}_{p}(A \square B) \leq$ $\mathbb{P}_{p}(A) \mathbb{P}_{p}(B)$.

We remark that van den Berg and Kesten conjectured that their inequality could be generalized to arbitrary sets; this remained open for almost a decade until it was resolved by Reimer [91]. 
The third result is specific to planar percolation. Russo [93] and Seymour and Welsh [101] proved that in an $m \times n$ discrete rectangle, the probability of an open crossing between the length- $m$ sides has a non-zero lower bound depending only on the aspect ratio $m / n$. The following is a straightforward consequence of their result:

Theorem 3.7. There exist positive constants $c, \lambda$ such that the $\mathbb{P}^{\delta}$-probability of a blue crossing of an annulus with inner radius $r$ and outer radius $R$ is $\lesssim(r / R)^{\lambda}$ provided $R / r, r / \delta \geq c$.

For proofs of the above results see $[23,47]$. A proof of Thm. 3.7 can also be found in $[117]$.

\subsection{Preharmonic functions}

We now arrive at the central argument of Smirnov's proof. By way of historical background Smirnov mentions the classical connection of Kakutani [57] between Brownian motion and harmonic functions described in $\S 2.2$. The key to Smirnov's proof was the discovery of a "preharmonic" function $s_{2}^{\delta}$ on $D_{2}^{\delta}$, the "separating probability function," which encodes the crossing probability. One then shows that as $\delta \downarrow 0, s_{2}^{\delta}$ converges to a harmonic function $s_{2}$ which encodes the limiting crossing probability. The result follows because $s_{2}$ is a conformal invariant.

The function $s_{2}^{\delta}$ is part of a triple $\left(s_{1}^{\delta}, s_{2}^{\delta}, s_{3}^{\delta}\right)$ which we now define: let $D_{4}^{\delta}$ be a four-marked domain, and let $D_{3}^{\delta}$ be the three-marked domain obtained by forgetting $P_{4}^{\delta}$. For $z \in D^{\delta} \cap H$ and $1 \leq j \leq 3$, let

$$
E_{j}(z) \equiv E_{j}\left(z ; D_{3}^{\delta}\right)
$$

denote the event that there is a blue $A_{j-1}-A_{j+1}$ simple path separating $z$ from $A_{j}$ (indices taken modulo 3). A schematic diagram is shown in Fig. 6. The separating probability functions for $D_{3}^{\delta}$ are

$$
s_{j}^{\delta}(z) \equiv \mathbb{P}^{\delta}\left[E_{j}(z)\right], \quad 1 \leq j \leq 3 .
$$

In particular, notice that $s_{2}^{\delta}\left(P_{4}^{\delta}\right)$ is exactly the crossing probability $\Phi^{\delta}\left(D_{4}^{\delta}\right)$. The plan is then to prove that $s_{2}^{\delta}$ converges to a function $s_{2}$ which is a conformal invariant of $D_{3}$. But $s_{2}\left(P_{4}\right)$ is the desired limiting crossing probability $\Phi\left(D_{4}\right)$, which must then be a conformal invariant.

\subsubsection{Cauchy-Riemann equations}

The approach is to take advantage of the relationships among the $s_{j}^{\delta}$ to show that they form a (discrete) "harmonic conjugate triple." This concept is easier to describe in the continuous setting: we will say that real-valued harmonic functions $h_{1}, h_{2}, h_{3}$ form a harmonic conjugate triple if $f=h_{1}+\zeta h_{2}+\zeta^{2} h_{3}$ is holomorphic, where $\zeta \equiv \zeta_{3} \equiv e^{2 \pi i / 3}$. 


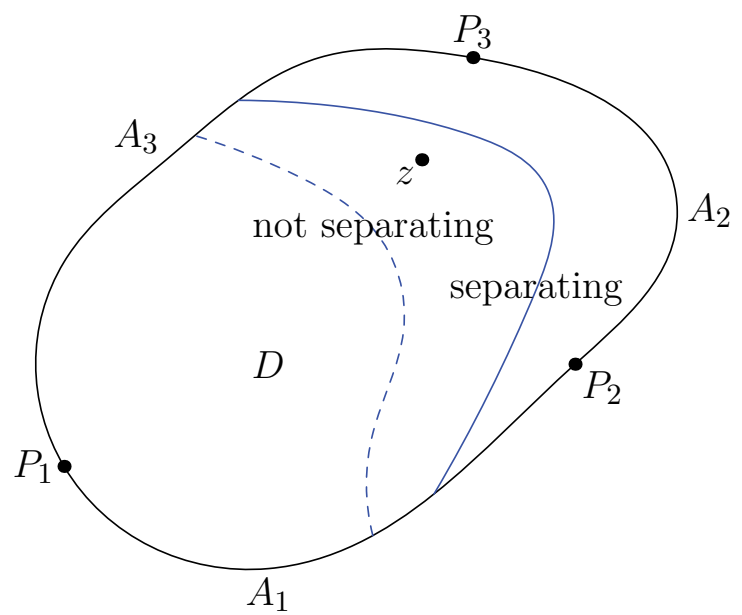

Fig 6: Schematic of separating event $E_{2}(z)$

Here is a version of the Cauchy-Riemann equations for harmonic conjugate triples: consider the directional derivatives

$$
\partial_{k} f(z) \equiv \lim _{h \rightarrow 0} \frac{f\left(z+h \zeta^{k-1}\right)-f(z)}{h}, \quad 1 \leq k \leq 3 .
$$

The same argument used in deriving the usual Cauchy-Riemann equations (see e.g. [113, p. 12]) gives

$$
\partial_{k} f=\frac{1}{\zeta} \partial_{k+1} f=\frac{1}{\zeta^{2}} \partial_{k+2} f,
$$

It will turn out that $h_{1}+h_{2}+h_{3} \equiv 1$, so the $\partial_{k} h_{j}$ are uniquely determined by the real linear relations

$$
\left(\begin{array}{c}
\partial_{k} \operatorname{Re} f \\
\partial_{k} \operatorname{Im} f \\
0
\end{array}\right)=\left(\begin{array}{ccc}
1 & \operatorname{Re} \zeta & \operatorname{Re} \zeta^{2} \\
1 & \operatorname{Im} \zeta & \operatorname{Im} \zeta^{2} \\
1 & 1 & 1
\end{array}\right)\left(\begin{array}{c}
\partial_{k} h_{1} \\
\partial_{k} h_{2} \\
\partial_{k} h_{3}
\end{array}\right) .
$$

Matching coefficients in (3.2) gives the $2 \pi / 3$-rotational Cauchy-Riemann equations

$$
\partial_{k} h_{j}=\partial_{k+1} h_{j+1}=\partial_{k+2} h_{j+2}, \quad 1 \leq k, j \leq 3,
$$

with indices taken modulo three.

We return now to the discrete setting. Recalling the definition (3.1), for $w \sim z$ in $\delta H \cap D^{\delta}$ define

$$
d_{z} s_{j}^{\delta}(w) \equiv \mathbb{P}^{\delta}\left[E_{j}(z) \backslash E_{j}(w)\right] .
$$

The following result is Smirnov's "color switching identity," and is a discrete version of the $2 \pi / 3$-rotational Cauchy-Riemann equations (3.3). 


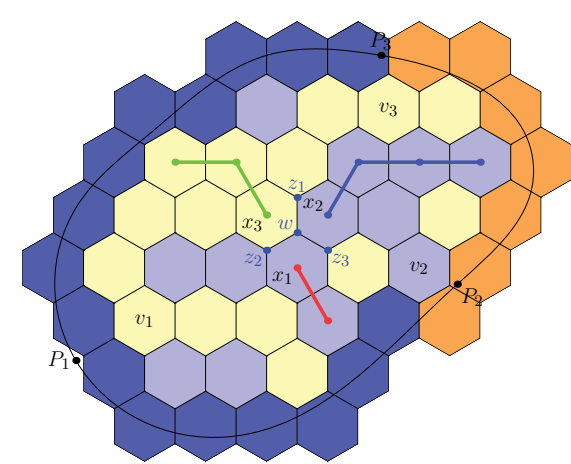

(a) $E_{3}^{\delta}\left(z_{3}\right) \backslash E_{3}^{\delta}(w)=B_{1} B_{2} Y_{3}$

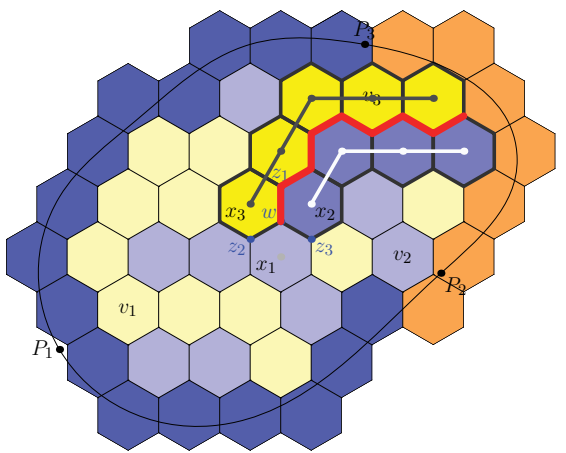

(b) Interface; "innermost" disjoint paths

Fig 7: Color switching

Proposition 3.8 (color switching). In the setting of Thm. 3.5, let $w \in \delta H \cap$ $D^{\delta}$, and fix a counterclockwise ordering $z_{1}, z_{2}, z_{3}$ of the neighbors of $w$ in $\delta H$, assumed to lie in $D^{\delta}$. Then

$$
d_{z_{k}} s_{j}^{\delta}(w)=d_{z_{k+1}} s_{j+1}^{\delta}(w)=d_{z_{k+2}} s_{j+2}^{\delta}(w) .
$$

Further $d_{z} s_{j}^{\delta}(w)=O\left(\delta^{\lambda}\right)$ where $\lambda$ is as in Thm. 3.\%.

Proof. For now we suppress the dependence on $\delta$ from the notation. By cyclic permutation of indices it suffices to prove the result with $k=j$. Let $x_{j} \in T$ be the center of the hexagon incident to $w$ opposite $z_{j}$, and let $C_{j}$ denote the event that there is a path $\eta_{j}$ of color $C \in\{B=$ blue, $Y=$ yellow $\}$ joining $x_{j}$ to $A_{j}$. Then

$$
E_{j}\left(z_{j}\right) \backslash E_{j}(w)=B_{j-1} Y_{j} B_{j+1},
$$

where the right-hand side denotes the event that $B_{j-1} \cap Y_{j} \cap B_{j+1}$ occurs via disjoint paths $\eta_{j-1}, \eta_{j}, \eta_{j+1}$. Fig. 7a illustrates this equality of events; a detailed proof of this statement may be found in [23, Ch. 7]. Thus (3.4) may be restated as

$$
\mathbb{P}\left(Y_{1} B_{2} B_{3}\right)=\mathbb{P}\left(B_{1} Y_{2} B_{3}\right)=\mathbb{P}\left(B_{1} B_{2} Y_{3}\right) .
$$

By symmetry it suffices to prove the second identity, which by flipping blue and yellow is the same as $\mathbb{P}\left(Y_{1} B_{2} Y_{3}\right)=\mathbb{P}\left(B_{1} B_{2} Y_{3}\right)$.

Conditioned on $B_{2} Y_{3}$, consider exploring the interface beginning at the edge separating $H_{x_{2}}$ (blue) from $H_{x_{3}}$ (yellow), oriented so that blue is to the right. This exploration path $P$ must end on some vertex of $H \cap\left(A_{2} \cup A_{3}\right)$, and the hexagons adjacent to $P$ determine the "innermost" disjoint paths $\eta_{2}$ and $\eta_{3}$ corresponding to $B_{2}$ and $Y_{3}$ respectively (erase loops so that the $\eta_{j}$ are simple paths). Note that these paths are determined without looking at any sites not incident to $P$.

Conditioning on the explored sites, the event $C_{1} B_{2} Y_{3}$ occurs if and only if there is a path of color $C$ from $x_{1}$ to $A_{1}$ which is disjoint from the explored 
sites. But the probability of this event clearly does not depend on whether $C$ is blue or yellow, so $\mathbb{P}\left(Y_{1} B_{2} Y_{3} \mid B_{2} Y_{3}\right)=\mathbb{P}\left(B_{1} B_{2} Y_{3} \mid B_{2} Y_{3}\right)$ and (3.4) is proved.

Finally, since the maximum distance from $w$ to an arc $A_{k}$ is of constant order, it follows from Thm. 3.7 that $d_{z} s_{j}^{\delta}(w)=O\left(\delta^{\lambda}\right)$.

Remark 3.9. In fact $d_{z} s_{j}^{\delta}(w)=O\left(\delta^{2 / 3}\right)$; see [108]. However, we will see that the $s_{j}^{\delta}$ converge to harmonic functions, which suggests that the discrete derivatives

$$
s_{j}^{\delta}(z)-s_{j}^{\delta}(w)=d_{z} s_{j}^{\delta}(w)-d_{w} s_{j}^{\delta}(z)
$$

are in fact $O(\delta)$ - that is, there is some cancellation between the disjoint events $E_{j}(z) \backslash E_{j}(w)$ and $E_{j}(w) \backslash E_{j}(z)$. It is unclear why this happens, and we turn now instead to proving relations among contour integrals, where the estimate of Propn. 3.8 is sufficient.

\subsubsection{Integral relations}

In taking the limit $\delta \downarrow 0$ it is easier to use global manifestations of holomorphicity such as Morera's theorem (see e.g. [113, Thm. 5.1]). In this section we use Propn. 3.8 to prove relations on discrete contour integrals.

Suppose $\left(h_{1}, h_{2}, h_{3}\right)$ form a harmonic conjugate triple: since $f$ and 1 are both holomorphic, by Morera's theorem

$$
\left(\begin{array}{l}
0 \\
0
\end{array}\right)=\left(\begin{array}{ccc}
1 & \zeta & \zeta^{2} \\
1 & 1 & 1
\end{array}\right)\left(\begin{array}{l}
I_{1} \\
I_{2} \\
I_{3}
\end{array}\right), \quad I_{j} \equiv \oint_{C} h_{j} d z,
$$

therefore $\oint_{C} h_{j+1} d z=\zeta \oint_{C} h_{j} d z$. It is instructive to note that this relation can alternately be derived from the $2 \pi / 3$-rotational Cauchy-Riemann equations (3.3), using Stokes's theorem. To see this, write

$$
d x_{1} \equiv d x, \quad d x_{2} \equiv-\frac{1}{2} d x+\frac{\sqrt{3}}{2} d y, \quad d x_{3} \equiv-\frac{1}{2} d x-\frac{\sqrt{3}}{2} d y .
$$

Then

$$
\begin{aligned}
& \sum_{k=1}^{3} \partial_{k} h_{j} d x_{k}=\frac{3}{2}\left[\partial_{x} h_{j} d x+\partial_{y} h_{j} d y\right]=\frac{3}{2} d h \\
& \sum_{k=1}^{3} \zeta^{k-1} d x_{k}=\frac{3}{2}[d x+i d y]=\frac{3}{2} d z
\end{aligned}
$$

Thus, for a contour $C=\partial U$, Stokes's theorem implies

$$
\oint_{C} h_{j} d z=\frac{4}{9} \int_{U}\left(\sum_{k=1}^{3} \partial_{k} h_{j} d x_{k}\right) \wedge\left(\sum_{k=1}^{3} \zeta^{k-1} d x_{k}\right) .
$$


Applying the Cauchy-Riemann equations (3.3) then gives

$$
\begin{aligned}
& \oint_{C} h_{j} d z=\frac{4}{9} \int_{U}\left(\sum_{k=1}^{3} \partial_{k+1} h_{j+1} d x_{k}\right) \wedge\left(\sum_{k=1}^{3} \zeta^{k-1} d x_{k}\right) \\
& =\frac{1}{\zeta} \cdot \frac{4}{9} \int_{U}\left(\sum_{k=1}^{3} \partial_{k} h_{j+1} d x_{k-1}\right) \wedge\left(\sum_{k=1}^{3} \zeta^{k-1} d x_{k-1}\right)=\frac{1}{\zeta} \oint_{C} h_{i+1} d z,
\end{aligned}
$$

the same relation derived above.

We will now prove a discrete analogue of this relation for certain contours; the proof will be a discrete version of the Stokes' theorem computation above. We will say that a discrete triangular contour is a contour which is a triangular path. For a triangular contour $C$ oriented counterclockwise in $D^{\delta}$, we define the discrete contour integral

$$
\oint_{C}^{\mathrm{d}} s_{j}^{\delta}(z) d z=\sum_{e \in C} s_{j}^{\delta}(z(e))\left(e^{+}-e^{-}\right)
$$

where $z(e)$ is the vertex of $H$ immediately to the left of the edge $e=\left(e^{-}, e^{+}\right)$.

Proposition 3.10. In the setting of Thm. 3.5, if $C$ is a discrete triangular contour in $D^{\delta}$ of length $L$, then

$$
\oint_{C}^{\mathrm{d}} s_{j+1}^{\delta}(z) d z-\zeta \oint_{C}^{\mathrm{d}} s_{j}^{\delta}(z) d z=O\left(\delta^{\lambda} L\right)
$$

where $\lambda$ is as in Thm. 3.7.

Proof. Let $C^{\circ}$ denote the points of $\delta H$ inside $C$, and use $1+\zeta+\zeta^{2}=0$ to write $\oint_{C}^{\mathrm{d}} s_{j}^{\delta}(z) d z=i \sqrt{3} \sum_{w \in C^{\circ}} \sum_{z \sim w, z \notin C^{\circ}} s_{j}^{\delta}(w)(z-w)=i \sqrt{3} \sum_{w \in C^{\circ}} \sum_{z \sim w, z \in C^{\circ}} s_{j}^{\delta}(w)(w-z)$.

This can be rewritten as

$$
\begin{aligned}
& \sum_{\{w, z\}}(z-w)\left[s_{j}^{\delta}(z)-s_{j}^{\delta}(w)\right]=\sum_{\{w, z\}}(z-w)\left[d_{z} s_{j}^{\delta}(w)-d_{w} s_{j}^{\delta}(z)\right] \\
& =\sum_{w \in C^{\circ}} \sum_{z \sim w} d_{z} s_{j}^{\delta}(w)(z-w)-\sum_{w \in C^{\circ}} \sum_{z \sim w, z \notin C^{\circ}} d_{z} s_{j}^{\delta}(w)(z-w) \equiv S_{j}-E_{j} .
\end{aligned}
$$

Propn. 3.8 implies $S_{j+1}=\zeta S_{j}$ while $E_{j}=O\left(\delta^{\lambda} L\right)$, which proves the result.

\subsection{Completing the proof}

We can easily extend $s_{j}^{\delta}$ to all of $\delta H$ by setting $s_{j}^{\delta}(z), z \notin D^{\delta}$, to equal $s_{j}^{\delta}\left(z^{\prime}\right)$ where $z^{\prime}$ is one of the hexagonal vertices inside $D^{\delta}$ closest to $z$. Let $g_{j}^{\delta}$ denote the extension to the closure of $D$ by linear interpolation. We will prove Thm. 3.5 by showing that the $g_{j}^{\delta}$ converge uniformly to the triple characterized by the following lemma, due to Beffara: 
Lemma 3.11 (Beffara [10]). Let $D_{3}$ be a three-marked domain. There is unique triple of continuous real-valued functions $\left(h_{1}, h_{2}, h_{3}\right)$ defined on the closure of $D$ satisfying the following two conditions:

(i) For any triangular contour $C$ in $D, \oint_{C} h_{j+1}(z) d z=\zeta \oint_{C} h_{j}(z) d z$.

(ii) For any $z \in A_{j}, h_{j}(z)=0$ and $h_{j+1}(z)+h_{j+2}(z)=1$.

Moreover these functions are harmonic, hence a conformal invariant of $D_{3}$.

Proof. Property (i) and Morera's theorem give that $f=h_{1}+\zeta h_{2}+\zeta^{2} h_{3}$ and $h=h_{1}+h_{2}+h_{3}$ are holomorphic, hence the $h_{j}$ are harmonic. Property (ii) and the maximum principle then gives $h \equiv 1$.

On the $\operatorname{arc} A_{j}$, property (ii) implies that $f$ is a convex combination of $\zeta^{j}$ and $\zeta_{j+1}$. Thus, as $z$ travels along $\partial D, f(z)$ winds around $\partial \Delta$ where $\Delta$ is the equilateral triangle with marked vertices $1, \zeta, \zeta^{2}$. It follows from the argument principle (see e.g. [113, Thm. 4.1]) that $f$ is unique conformal equivalence of $D_{3}$ with $\Delta$. The result follows since the $h_{j}$ are uniquely determined by $(f, h)$.

Proof of Thm. 3.5. It is clear that the $g_{j}^{\delta}$ are uniformly bounded, and uniform equicontinuity follows from the bound on the discrete derivatives (Propn. 3.8), so the $g_{j}^{\delta}$ have subsequential uniform limits by the Arzelà-Ascoli theorem. We must show that any subsequential limit satisfies the characterization of Lem. 3.11. Indeed, property (i) follows from Propn. 3.10 by approximating $C$ with discrete triangular contours, using uniform convergence to the limit and the uniform equicontinuity of the $g_{j}^{\delta}$. Property (ii) is intuitively clear, and is easy to prove rigorously using Thm. 3.7.

It follows that $\Phi^{\delta}\left(D_{4}^{\delta}\right)=g_{2}^{\delta}\left(P_{4}^{\delta}\right)$ converges to $\Phi\left(D_{4}\right)=h_{2}\left(P_{4}\right)$, which is a conformal invariant of $D_{4}$ by Lem. 3.11. This concludes the proof of Smirnov's theorem.

Carleson noted that Cardy's formula takes a particularly simple form when the domain is an equilateral triangle. A consequence of the above proof is the verification of this formula for critical percolation on $T$ :

Cardy's formula in Carleson's form (Smirnov [105, 108], Beffara [10]). Let $D_{3}$ denote the three-marked domain obtained from $D_{4}$ by forgetting $P_{4}$, and $f$ the conformal equivalence $D_{3} \rightarrow \Delta$. Then $\Phi\left(D_{4}\right)=x\left(D_{4}\right)$ where $x \equiv x\left(D_{4}\right)$ is defined by

$$
f\left(P_{4}\right)=x \zeta^{2}+(1-x) .
$$

Proof. For $x \in A_{3}(\Delta)$ write $\Delta_{x} \equiv\left(\Delta ; 1, \zeta, \zeta^{2}, x\right)$ : Thm. 3.5 implies

$$
\Phi\left(D_{4}\right)=\Phi\left(\Delta_{f\left(P_{4}\right)}\right)=h_{2}\left(f\left(P_{4}\right)\right)
$$

where $\left(h_{1}, h_{2}, h_{3}\right)$ is the triple on $\Delta$ given by Lem. 3.11. But $h_{1}+\zeta h_{2}+\zeta^{2} h_{3}$ must simply be the identity on $\Delta$, so $h_{2}$ is linear on $A_{3}(\Delta)$ and the result follows.

In forthcoming work Mendelson, Nachmias, Sheffield and Watson prove an $O\left(\delta^{\epsilon}\right)$ bound on the rate of convergence to Cardy's formula [83].

For two more percolation formulas of a similar nature see [40, 95, 104]. 


\subsection{The percolation exploration path}

Let $D_{2}^{\delta} \equiv\left(D^{\delta} ; a^{\delta}, b^{\delta}\right)$ be a two-marked domain, and suppose $A_{1}^{+}, A_{2}^{+}$are colored yellow, blue respectively. The percolation exploration path $\gamma^{\delta}$ is the portion of the blue-yellow interface traveling from $a^{\delta}$ to $b^{\delta}$. The purpose of the remainder of this article is to prove the following:

Theorem 3.12 (Schramm [94], Smirnov [105, 108], Camia and Newman [31]). Let $\gamma^{\delta}$ denote the percolation exploration path in the discrete domain $D_{2}^{\delta}$. If $D_{2}^{\delta}$ converges uniformly to the two-marked bounded Jordan domain $D_{2}$, then $\gamma^{\delta}$ converges weakly to chordal $S L E_{6}$ in $D_{2}$.

Remark 3.13. For random variables on a separable metric space, there are several equivalent ways to define convergence in law (weak convergence, convergence in distribution): in our setting, a natural formulation is via the Skorohod coupling theorem (more precisely, the generalization due to Dudley [42]) which states that random variables converge in law if and only if they can be defined on a joint probability space in which they converge almost surely.

In $\S 4$ we define SLE and give some characterizations of $\mathrm{SLE}_{6}$. $\S 5$ shows that the $\gamma^{\delta}$ have subsequential weak limits. Finally, $\S 6$ shows that all subsequential limits coincide with chordal $\mathrm{SLE}_{6}$, concluding the proof.

\section{Schramm-Loewner evolutions}

In this chapter we derive the chordal Loewner differential equation

$$
\dot{g}_{t}(z)=\frac{2}{g_{t}(z)-u_{t}}, \quad g_{0}(z)=z, \quad u_{t}=g_{t}(\gamma(t))
$$

for a self-avoiding (see Defn. 4.8 below) curve traveling from 0 to $\infty$ in $\overline{\mathbb{H}}$. We then show that the SLE axioms imply that $u_{t}$ is a Brownian motion. We conclude with a characterization of $\mathrm{SLE}_{6}$ which will be used in the proof of Thm. 3.12. A fully rigorous treatment of SLE is beyond the scope of this article, but we attempt to highlight the main points.

\subsection{Normalizing the conformal maps}

By conformal invariance it suffices to define chordal SLE for a single two-marked domain, and it turns out a convenient choice is $\mathbb{H}_{2} \equiv(\mathbb{H} ; 0, \infty)$. In this section only, we take $\mathbb{C}$ with the spherical metric, so that $\mathbb{H}$ is a bounded domain with compact closure $\overline{\mathbb{H}}=\mathbb{H} \cup \overline{\mathbb{R}}$. We will begin by describing the Loewner slit mapping theorem for a simple curve $\gamma$ traveling chordally in $\mathbb{H}_{2}$, and extend to more general curves afterwards.

Recall that conformal automorphisms of a simply connected domain are determined up to three real degrees of freedom, for example, the group of conformal automorphisms of $\mathbb{H}$ is the group of Möbius transforms, Aut $\mathbb{H} \cong \mathrm{SL}_{2} \mathbb{R}$ (see e.g. 
[113, Thm. 2.4]). Thus conformal equivalences between two-marked domains are not uniquely determined, so we must choose an appropriate normalization for the maps $g_{t}$.

From now on we write $f \lesssim g$ if $f / g$ is bounded by a (universal) constant, and $f \asymp g$ if both $f \lesssim g$ and $g \lesssim f$.

\subsubsection{Boundary behavior}

We begin with a caution that while conformal maps are as nice as possible in the interiors of domains, their good behavior does not necessarily continue up to the boundary. The results of this section may be found in [90] which has much more information on this subject.

Carathéodory's theorem. Let $f: \mathbb{D} \rightarrow D$ be a conformal map. The function $f$ has a continuous extension to $\overline{\mathbb{D}}$, which restricts to a bijection of $C=\partial \mathbb{D}$ with $\partial D$, if and only if $\partial D$ is a Jordan curve.

Unfortunately, Carathéodory's theorem does not apply in our main case of interest, where $D$ is a "slit" domain of the form $\mathbb{H} \backslash \gamma[0, t]$. We do, however, have the following result:

Definition 4.1. The closed set $A \subseteq \mathbb{C}$ is called locally connected if for all $\epsilon>0$ there exists $\delta>0$ such that, for any two points $a, b \in A$ with $|a-b|<\delta$, we can find a continuum ${ }^{7} B \subseteq A$ with $a, b \in B$ and $\operatorname{diam} B<\epsilon$.

Continuity theorem. Let $f: \mathbb{D} \rightarrow D$ be a conformal map. The following are equivalent:

(i) The function $f$ has a continuous extension to $\overline{\mathbb{D}}$;

(ii) $\partial D$ is a curve;

(iii) $\partial D$ is locally connected;

(iv) $\mathbb{C} \backslash D$ is locally connected.

This tells us that the maps are well-behaved at least in one direction, and in the other direction more care is needed. In particular, the driving function " $u_{t}=g_{t}(\gamma(t))$ " is not a priori well-defined.

\subsubsection{Half-plane capacity}

We define a compact $\mathbb{H}$-hull to be a bounded subset $A \subset \mathbb{H}$ with $A$ closed in $\mathbb{H}$ and $\mathbb{H} \backslash A$ simply connected ( $A$ itself is not required to be connected). The radius of $A$, denoted $\operatorname{rad} A$, is the radius of the smallest closed disc centered at the origin which contains $A$. Let $\mathcal{Q}$ denote the collection of compact $\mathbb{H}$-hulls.

We will normalize a conformal map $g_{A}: \mathbb{H} \backslash A \rightarrow \mathbb{H}$ by the requirement that it behave "like the identity" near $\infty$. The following proposition makes this precise:

\footnotetext{
${ }^{7} \mathrm{~A}$ nonempty compact connected subset of $\mathbb{C}$.
} 
Proposition 4.2. For $A \in \mathcal{Q}$, there is a unique conformal transformation $g_{A}: \mathbb{H} \backslash A \rightarrow \mathbb{H}$ such that

$$
\lim _{z \rightarrow \infty}\left[g_{A}(z)-z\right]=0 .
$$

We say that $g_{A}$ has the hydrodynamic normalization.

Proof. The inversion map $j: z \mapsto-1 / z$ is an element of Aut $\mathbb{H} . D=j(\mathbb{H} \backslash A) \subseteq$ $\mathbb{H}$ is simply connected and contains the intersection of $\mathbb{H}$ with some neighborhood of 0. By the Riemann mapping theorem and Carathéodory's theorem, there exists a conformal map $\phi: D \rightarrow \mathbb{H}$ with $\phi(0)=0$, extending continuously to the boundary of $D$ near 0. By Schwarz reflection (see $[1, \S 4.6 .5]$ ) $\phi$ has a power series expansion

$$
\phi(z)=a_{1} z+a_{2} z^{2}+a_{3} z^{3}+\cdots
$$

around 0 with $a_{1}>0$ and $a_{k} \in \mathbb{R}$ for all $k$, since $\phi$ maps the real line near 0 to the real line. The map $\phi$ is determined up to composition with any element of Aut $\mathbb{H} \cong \mathrm{SL}_{2} \mathbb{R}$ fixing 0 , and a simple calculation shows that $\phi$ is fully determined by the choice of $a_{1}>0, a_{2} \in \mathbb{R}$. Define $g_{A}(z) \equiv j \circ \phi \circ j(z)$; its expansion around $\infty$ is

$$
g_{A}(z) \equiv \frac{-1}{\phi(-1 / z)}=\frac{z}{a_{1}}+\frac{a_{2}}{a_{1}^{2}}+\left(\frac{a_{2}^{2}}{a_{1}^{3}}-\frac{a_{3}}{a_{1}^{2}}\right) \frac{1}{z}+\cdots \equiv b_{-1} z+b_{0}+\frac{b_{1}}{z}+\cdots .
$$

The result follows by choosing $a_{1}=1, a_{2}=0$.

Definition 4.3. If $A$ is a compact $\mathbb{H}$-hull, the half-plane capacity (from $\infty$ ) of $A$ is

$$
\text { hcap } A=\lim _{z \rightarrow \infty} z\left[g_{A}(z)-z\right] \text {. }
$$

In the notation of the proof of Propn. 4.2, hcap $A=b_{1}$ and

$$
g_{A}(z)=z+\frac{\operatorname{hcap} A}{z}+O\left(\frac{1}{|z|^{2}}\right) \text { as } z \rightarrow \infty .
$$

If $r>0$ then $g_{r A}(z)=r g_{A}(z / r)$ so hcap $(r A)=r^{2}$ hcap $A$, and if $x \in \mathbb{R}$ then $g_{A+x}=g_{A}(z-x)+x$ so hcap $(A+x)=$ hcap $A$. If $A, B \in \mathcal{Q}$ with $A \subseteq B$ then $g_{B}=g_{g_{A}(B \backslash A)} \circ g_{A}$, and expanding gives

$$
\text { hcap } B=\operatorname{hcap} A+\operatorname{hcap}\left(g_{A}(B \backslash A)\right) \text {. }
$$

This suggests that the half-plane capacity is a measure of the size of $A$, but we have not yet shown that hcap is positive. The following result proves this and gives a more precise characterization of the capacity in terms of Brownian motion.

Proposition 4.4. Let $A \in \mathcal{Q}, W_{t}$ a Brownian motion started at $z \in \mathbb{H} \backslash A$, and $\tau \equiv \tau_{\mathbb{H} \backslash A}$ the leaving time of $\mathbb{H} \backslash A$. Then $\operatorname{Im}\left[z-g_{A}(z)\right]=\mathbb{E}_{z}\left(\operatorname{Im} W_{\tau}\right)$. 
Proof. By the hydrodynamic normalization, $\phi(z)=\operatorname{Im}\left[z-g_{A}(z)\right]$ is a bounded harmonic function on $\mathbb{H} \backslash A$, and can be extended continuously to the boundary by setting $\phi(z)=\operatorname{Im} z$ for $z \in \partial(\mathbb{H} \backslash A)$. Therefore $\phi\left(W_{t \wedge \tau}\right)$ is a martingale, and the optional sampling theorem gives

$$
\phi(z)=\mathbb{E}_{z} \phi\left(W_{\tau}\right)=\mathbb{E}_{z}\left[\operatorname{Im} W_{\tau}\right],
$$

proving the result.

An immediate consequence is that

$$
\text { hcap } A=\lim _{y \rightarrow \infty} y \mathbb{E}_{i y}\left[\operatorname{Im} W_{\tau}\right],
$$

which proves that hcap is positive. For example, $g_{\overline{\mathbb{D}} \cap \mathbb{H}}(z)=z+z^{-1}$ so hcap $(\overline{\mathbb{D}} \cap$ $\mathbb{H})=1$, implying the general bound hcap $A \leq(\operatorname{rad} A)^{2}$. For more geometric interpretations of hcap see [68].

We now state two results which will be used in the LDE derivation; the proofs are deferred to $\S 4.4$. The first result is a uniform estimate on higherorder remainder terms in the Laurent expansion of $g_{A}(z)$ :

Proposition 4.5. There exists a constant $c<\infty$ such that

$$
\left|g_{A}(z)-z-\frac{\operatorname{hcap} A}{z}\right| \leq c \frac{(\operatorname{rad} A)(\operatorname{hcap} A)}{|z|^{2}}
$$

for all $|z| \geq 2 \operatorname{rad} A$.

The next result is a bound on the distortion of size under the maps $g_{A}$.

Proposition 4.6. For $A \in \mathcal{Q}$ and $B \subset \mathbb{H}$ such that $A \cup B \in \mathcal{Q}$,

$$
\operatorname{diam} g_{A}(B) \lesssim[(\operatorname{diam} B)(\sup \{\operatorname{Im} z: z \in B\})]^{1 / 2} .
$$

Assuming these results, in the next section we derive the LDE and use it to define the Schramm-Loewner evolutions.

\subsection{The Loewner differential equation and $S L E$}

Returning to the original problem, let $\gamma$ be a simple curve traveling from 0 to $\infty$ in $\mathbb{H}$. Let

$$
g_{t} \equiv g_{\gamma(0, t]}, \quad b(t) \equiv \operatorname{hcap} \gamma(0, t] .
$$

Let $g_{s, t} \equiv g_{g_{s}(\gamma(s, t])}$ so that $g_{t}=g_{s, t} \circ g_{s}$. The following is a consequence of Propn. 4.6:

Corollary 4.7. The map $g_{t}$ can be extended continuously to $u_{t} \equiv g_{t}(\gamma(t))$ which is real-valued and continuous in $t$ with $u_{0}=0$. 
Proof. Note that

$$
\left\|g_{s}-g_{t}\right\|_{\infty}=\sup _{z}\left|g_{s, t}(z)-z\right| \lesssim \operatorname{diam} g_{s}(\gamma(s, t]),
$$

and Propn. 4.6 implies

$$
\operatorname{diam} g_{s}(\gamma(s, t]) \lesssim[(\operatorname{diam} \gamma[s, t])(\operatorname{diam} \gamma[0, t])]^{1 / 2}
$$

Let $w_{t} \equiv \lim _{s \uparrow t} g_{s}(\gamma(t))$; (4.4) and (4.5) imply that $w$ is well-defined and continuous in $t$. Given $\epsilon>0$, since $\gamma$ is simple, there exists $0<\delta<\epsilon$ such that if $0 \leq s<t \leq t_{0}$ and $|\gamma(s)-\gamma(t)|<\delta$, then $t-s<\epsilon$ and $\operatorname{diam} \gamma[s, t]<\epsilon$. Let $B=B_{\delta}(\gamma(t)) \cap(\mathbb{H} \backslash \gamma[0, t])$ : for any $z \in B$, there exists a simple curve $\tilde{\gamma}$ in $B$ of diameter $<\delta$ joining $z$ to some $\gamma\left(t^{\prime}\right) \in B_{\delta}(\gamma(t))$ with $t^{\prime}<t$, hence $t-t^{\prime}<\epsilon$ and $\operatorname{diam} \gamma\left[t^{\prime}, t\right]<\epsilon$. Then

$$
\begin{aligned}
\left|g_{t}(z)-w_{t}\right| & \leq\left|g_{t}(z)-g_{t^{\prime}}(z)\right|+\left|g_{t^{\prime}}(z)-g_{t^{\prime}}(\gamma(t))\right|+\left|g_{t^{\prime}}(\gamma(t))-w_{t}\right| \\
& \leq\left\|g_{t}-g_{t^{\prime}}\right\|_{\infty}+\operatorname{diam} g_{t^{\prime}}(\tilde{\gamma})+\underset{s \uparrow t}{\limsup }\left\|g_{t^{\prime}}-g_{s}\right\|_{\infty},
\end{aligned}
$$

which by (4.4) and (4.5) can be made arbitrarily small by taking $\epsilon \downarrow 0$. Therefore $u_{t}=w_{t}$ is continuous in $t$.

By (4.1) and (4.5), the set $A=g_{s}(\gamma(s, t])-u_{s}$ has half-plane capacity $b(t)-$ $b(s)$ and radius $r_{s, t}$ which both approach zero as $t-s \downarrow 0$. By reparametrizing, suppose $b$ is continuously differentiable. Propn. 4.5 gives

$$
\begin{aligned}
& g_{t}(z)-u_{s}=g_{g_{s}(\gamma(s, t])-u_{s}}\left(g_{s}(z)-u_{s}\right) \\
& =\left[g_{s}(z)-u_{s}\right]+\frac{b(t)-b(s)}{g_{s}(z)-u_{s}}+O\left(\frac{r_{s, t}[b(t)-b(s)]}{\left|g_{s}(z)-u_{s}\right|^{2}}\right),
\end{aligned}
$$

where the final term tends to zero faster than $t-s$ as $t-s \downarrow 0$. Rearranging and taking $t-s \downarrow 0$ gives

$$
\dot{g}_{t}(z)=\frac{\dot{b}(t)}{g_{t}(z)-u_{t}}, \quad g_{0}(z)=z .
$$

The standard parametrization ${ }^{8}$ then sets $b(t)=2 t$, so that

$$
\dot{g}_{t}(z)=\frac{2}{g_{t}(z)-u_{t}}, \quad g_{0}(z)=z .
$$

This is the (chordal) Loewner differential equation (LDE) with driving function $u_{t}$.

In fact, the only place in the derivation where we used that $\gamma$ was simple was in the proof of Cor. 4.7. In fact, using Propn. 4.6 one can see that the LDE applies to any continuously increasing hull process: a strictly increasing process $K_{t} \in \mathcal{Q}$ with $b(t)=$ hcap $K_{t}$ continuously differentiable, such that $g_{t}\left(K_{t+\delta}\right)$ 


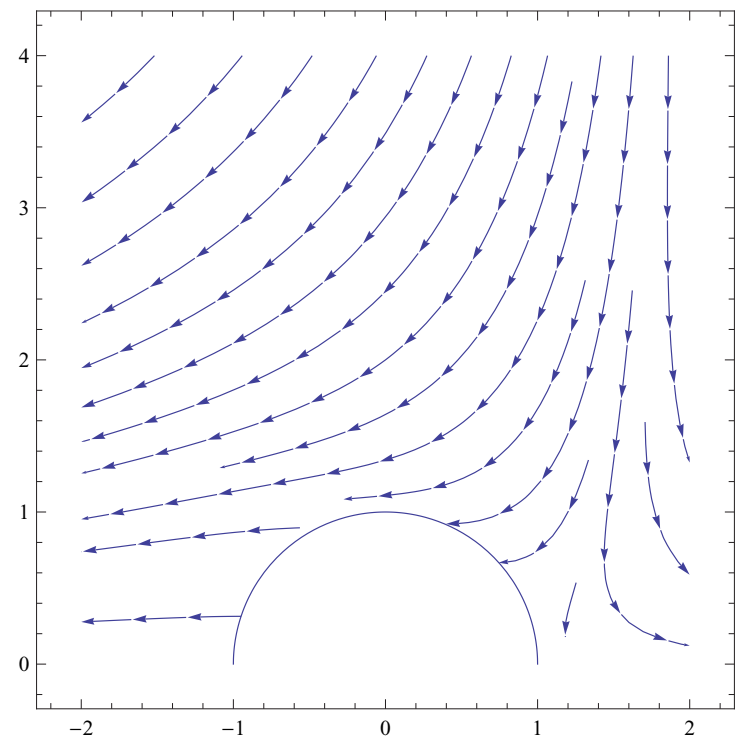

Fig 8: Vector field $2 /\left[g_{\mathbb{D}}(z)-2\right]$ around $\mathbb{D}$

decreases to a single point $u_{t} \in \mathbb{R}$ as $\delta \downarrow 0$ and $u_{t}$ is continuous in $t$. Fig. 8 shows the vector field $2 /\left[g_{\mathbb{D}}(z)-2\right]$ (corresponding to $K_{t}=\mathbb{D}$ and $u_{t}=2$ ).

Conversely, given a continuous function $u_{t}:[0, \infty) \rightarrow \mathbb{R}$ with $u_{0}=0$, the LDE with driving function $u_{t}$ is simply an ODE, so we can solve this ODE to recover the increasing hull process: $g_{t}(z)$ is well-defined up to the first time $T_{z}$ that $\lim _{t \uparrow T_{z}}\left[g_{t}(z)-u_{t}\right]=0$. Consequently $K_{t}=\left\{z \in \mathbb{H}: T_{z} \leq t\right\}$, the set of points "swallowed" by time $t$ - note that multiple points can be swallowed at once.

Definition 4.8. Let $\gamma$ be any curve (not necessarily simple) traveling from 0 to $\infty$ in $\overline{\mathbb{H}}$. The filling of $\gamma[0, t]$ is the set $K_{t} \in \mathcal{Q}$ of all $z \in \mathbb{H}$ not belonging to the unbounded component of $\mathbb{H} \backslash \gamma[0, t]$. We call $K_{t}$ the hull process generated by $\gamma$, and say that $\gamma$ is self-avoiding if $K_{t}$ is continuously increasing.

By abuse of notation we write hcap $\gamma[0, t] \equiv$ hcap $K_{t}$ : if $\gamma$ is self-avoiding then it has a parametrization with hcap $\gamma[0, t]=2 t$, in which case we say $\gamma$ is "parametrized by its half-plane capacity." If a curve travels for a non-trivial time interval into its past hull or along the domain boundary, its half-plane capacity remains constant during this time so it is not parametrized by hcap. There are also curves which may be parametrized by hcap but which do not generate a continuously increasing hull process, specifically curves with transversal selfcrossings.

If the (hypothetical) random curve $\gamma$ is to satisfy the SLE axioms, its driving function must be a continuous process of stationary independent increments. By

\footnotetext{
${ }^{8}$ For a discussion of parametrization of SLE see [78].
} 
Lévy's characterization of Brownian motion this implies $u_{t}=\mu t+\sqrt{\kappa} W_{t}$, for $W_{t}$ a standard Brownian motion. If further the model has left-right symmetry then $\mu=0$.

Definition 4.9. The Schramm-Loewner evolution on $\mathbb{H}_{2}$ with parameter $\kappa$ is the continuously increasing hull process given by the Loewner evolution (4.6) with random driving function $u_{t}=\sqrt{\kappa} W_{t}$.

The $\mathrm{SLE}_{\kappa}$ on an arbitrary two-marked domain $D_{2}$ is the image of $\mathrm{SLE}_{\kappa}$ on $\mathbb{H}_{2}$ under a conformal equivalence $f: \mathbb{H}_{2} \rightarrow D_{2}$ (and hence is defined only up to a linear time change). More generally, the $\mathrm{SLE}_{\kappa, \rho}$ processes are generated by Brownian motion with drift; see [39, 71, 100].

Unfortunately, there are continuously increasing hull process $K_{t}$ not generated by any curve (see e.g. $[72, \S 4.4]$ ). That the hull process corresponding to $u_{t}=\sqrt{\kappa} W_{t}$ is almost surely generated by a curve was proved for $\kappa=8$ in [77] and for $\kappa \neq 8$ in [92] (see [72, Ch. 7]). We do not need this fact because we will show that the percolation exploration path converges in law to a conformally invariant random curve satisfying a property which uniquely characterizes $\mathrm{SLE}_{6}$ among the $\mathrm{SLE}_{\kappa}$ (hull) processes. This implies a fortiori that $\mathrm{SLE}_{6}$ is generated by a curve.

\subsection{Characterization of $S L E_{6}$}

We now present the characterization of $\mathrm{SLE}_{6}$ used by Camia and Newman in identifying the scaling limit of the exploration path.

First, the following result, noted by Schramm in [94, §1] and proved in [73], indicates why $\mathrm{SLE}_{6}$ is the natural candidate. Let $N=\mathbb{H} \cap N_{0}$ for $N_{0}$ an open neighborhood of 0 , and let $\Phi$ be a locally real conformal map of $N$ into $\mathbb{H}$ (that is, in some neighborhood of $0, \Phi$ has a power series expansion with real coefficients). Consider running an $\mathrm{SLE}_{\kappa}$ process $\gamma$ until the first time $\tau$ that it leaves $N$ : the process is said to have the locality property if $\Phi \gamma$ (before time $\tau)$ again has the law of $\mathrm{SLE}_{\kappa}$ up to a time change.

Theorem 4.10 (Lawler, Schramm, Werner [71, 73]). The only $S L E_{\kappa}$ with the locality property is $S L E_{6}$.

Proof. We follow the proof of [72]. For $\gamma$ an $\mathrm{SLE}_{\kappa}$ process, let $K_{t}$ denote the filling of $\gamma[0, t]$, and let $N^{*}=\Phi N, \gamma^{*}=\Phi \gamma, K_{t}^{*}=\Phi K_{t}, b^{*}(t)=$ hcap $\gamma^{*}[0, t]$. Let $g_{t}^{*}$ denote the conformal map $\mathbb{H} \backslash K_{t}^{*} \rightarrow \mathbb{H}$ with the hydrodynamic normalization, and let $\Phi_{t} \equiv g_{t}^{*} \circ \Phi \circ g_{t}^{-1}$, so that

$$
\dot{g}_{t}^{*}(z)=\frac{\dot{b}^{*}(t)}{g_{t}^{*}(z)-u_{t}^{*}}, \quad g_{0}^{*}(z)=z,
$$

with $u_{t}^{*}=\Phi_{t}\left(u_{t}\right)$.

We leave it to the reader to verify that $\dot{b}^{*}(t)=2 \Phi_{t}^{\prime}\left(u_{t}\right)^{2}$. Therefore, we can easily calculate that for $z \in \mathbb{H}$,

$$
\dot{\Phi}_{t}(z)=2\left[\frac{\Phi_{t}^{\prime}\left(u_{t}\right)^{2}}{\Phi_{t}(z)-u_{t}^{*}}-\frac{\Phi_{t}^{\prime}(z)}{z-u_{t}}\right] .
$$


Letting $z \rightarrow u_{t}$, we find $\dot{\Phi}_{t}\left(u_{t}\right)=-3 \Phi^{\prime \prime}\left(u_{t}\right)$. Thus, by Itō's lemma,

$$
\begin{aligned}
d u_{t}^{*} & =\dot{\Phi}_{t}\left(u_{t}\right) d t+\Phi_{t}^{\prime}\left(u_{t}\right) d u_{t}+\frac{1}{2} \Phi_{t}^{\prime \prime}\left(u_{t}\right) \kappa d t \\
& =\left[-3+\frac{\kappa}{2}\right] \Phi_{t}^{\prime \prime}\left(u_{t}\right) d t+\Phi_{t}^{\prime}\left(u_{t}\right) d u_{t},
\end{aligned}
$$

which is a martingale if and only if $\kappa=6$. In this case it follows that $\gamma^{*}$ is again an $\mathrm{SLE}_{6}$ process.

Any scaling limit of the percolation exploration path is certainly expected to have the locality property, so (heuristically) Thm. 4.10 already distinguishes $\mathrm{SLE}_{6}$ as the only possible candidate for the scaling limit of the exploration path.

To rigorously identify the scaling limit with $\mathrm{SLE}_{6}$ we take a slightly different approach: recall $\S 2.2$ where we noted that from the conformal invariance of the Brownian hitting distribution, one can deduce the conformal invariance of the entire Brownian trajectory (up to reparametrization) by taking polygonal approximations. We now apply this idea to give a characterization of $\mathrm{SLE}_{6}$ which we will then verify in the percolation scaling limit.

By conformal invariance it suffices to characterize $\mathrm{SLE}_{6}$ in $\mathbb{H}_{2}$. For $\gamma$ any self-avoiding curve traveling chordally in $\mathbb{H}_{2}$ and parametrized by hcap, define

$$
\tau(\gamma, \epsilon) \equiv \inf \left\{t \geq 0: \gamma(t) \notin B_{\epsilon}\right\} ;
$$

note $\tau(\gamma, \epsilon) \leq \epsilon / 2$. Let $\tau_{0}=0$, and for $j \geq 1$ let

$$
\tau_{j}=\tau_{j-1}+\tau\left(\bar{g}_{\tau_{j-1}} \gamma, \epsilon\right), \quad \bar{g}_{t}(z) \equiv g_{t}(z)-u_{t} .
$$

Define the polygonal approximation $\gamma_{\epsilon}$ by setting $\gamma_{\epsilon}\left(\tau_{j}\right)=\gamma\left(\tau_{j}\right)$ and interpolating linearly in between. Since $\gamma$ is continuous on $[0, \infty]$ it is uniformly continuous, and since $\tau_{j}-\tau_{j-1} \leq \epsilon / 2$ for all $j$ it follows that $\gamma_{\epsilon} \rightarrow \gamma$ uniformly as $\epsilon \downarrow 0$. Thus a deterministic $\gamma$ is characterized by its polygonal approximations $\gamma_{\epsilon}$, and a random $\gamma$ is characterized by the laws of these approximations.

For fixed $\epsilon>0$, call $\widetilde{K}_{j} \equiv \bar{g}_{\tau_{j-1}} K_{\tau_{j}}(j \geq 1)$ the $\epsilon$-filling sequence for $\gamma$. Then $\bar{g}_{\tau_{j-1}} \gamma_{\epsilon}\left(\tau_{j}\right)$ is the unique point of intersection of $\widetilde{K}_{j}$ with $C_{\epsilon}$, and by composition of conformal mappings $\gamma_{\epsilon}$ is determined by the $\epsilon$-filling sequence. But if $\gamma$ is a random SLE curve, the domain Markov property implies that its $\epsilon$-filling sequence is i.i.d., characterized by the law of $\widetilde{K}_{1}$.

$\mathrm{SLE}_{6}$ is the unique $\mathrm{SLE}_{\kappa}$ for which this law is given by Cardy's formula, another fact noted in $[94, \S 1]$ and proved in [73]. We will formulate this result as follows: in a 2-marked domain $D_{2} \equiv(D ; a, b)$ let $J$ be a simple curve joining $c \in \overline{a b}, d \in \overline{b a}$. We refer to $J$ as a crosscut; it separates $D$ into subdomains $D_{0}$ (incident to $a$ ) and $D_{\infty}$ (incident to $b$ ), and we consider running a self-avoiding curve from $a$ until it exits $D_{0}$, resulting in a hull $K\left(D_{2}, J\right)$. Let $\alpha_{c}$ be a simple curve traveling from $\alpha_{c 0} \in \overline{a c}$ to $\alpha_{c 1} \in J$, and let $\alpha_{d}$ be a simple curve traveling from $\alpha_{d 0} \in \overline{d a}$ to $\alpha_{d 1} \in J$, with $\alpha_{c} \cap \alpha_{d}=\varnothing$. Let $D_{0}^{\alpha}$ denote the connected component of $D_{0} \backslash\left(\alpha_{c} \cup \alpha_{d}\right)$ between $\alpha_{c}$ and $\alpha_{d}$. (See Fig. 9.) Specifying the law of the hull $K$ is equivalent to specifying the law of its boundary, regarded as an 


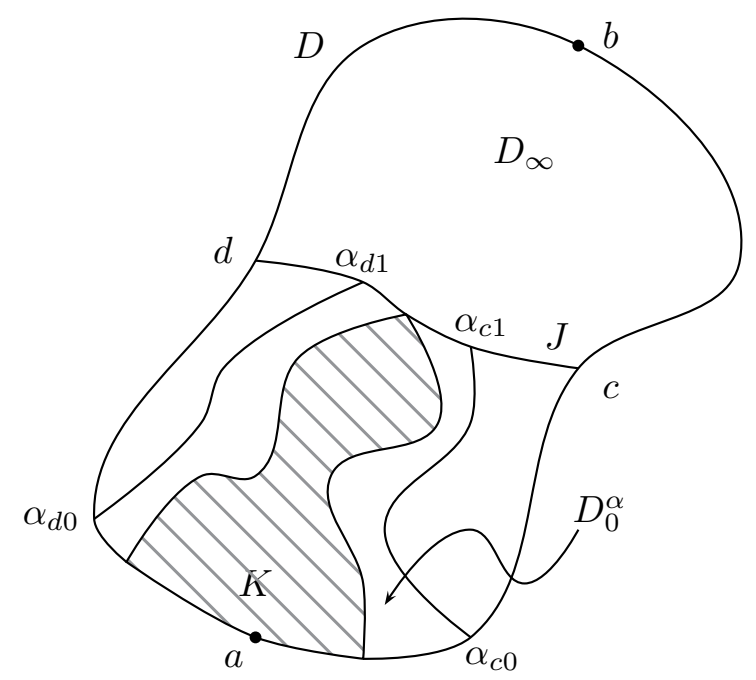

Fig 9: Crosscut and hull distribution

element of $\mathcal{C}_{D}$. Excluding events of probability zero, the $\sigma$-algebra is generated by the events

$$
E_{\alpha} \equiv\left\{K: K \subseteq \overline{D_{0}^{\alpha}}\right\}
$$

Theorem 4.11 (Lawler, Schramm, Werner [73, Thm. 3.2]). For the setting described above, $S L E_{6}$ is the unique $S L E_{\kappa}$ for which the law of $K\left(D_{2}, J\right)$ is determined by Cardy's formula:

$$
\mathbb{P}^{S L E_{6}}\left(E_{\alpha}\right)=\Phi\left(D_{0}^{\alpha} ; a, \alpha_{c 0}, \alpha_{c 1}, \alpha_{d 0}\right)-\Phi\left(D_{0}^{\alpha} ; a, \alpha_{c 0}, \alpha_{c 1}, \alpha_{d 0}\right) .
$$

We omit the proof and refer the reader to [73] and $[72, \S 6.7]$. The proof for the equilateral triangle (which is sufficient for our purposes) is particularly simple and may be found in $[72, \S 6.8]$ or $[117, \S 3.8]$.

The main consequence of this discussion is the following result which will be used in $\S 6$ to identify the scaling limit of the exploration path.

Proposition 4.12. If $\gamma$ is a self-avoiding curve such that for all $\epsilon>0$ the $\epsilon$-filling sequence is i.i.d. with law given by Cardy's formula (4.7), then $\gamma$ is $S L E_{6}$.

\subsection{Capacity estimates for the LDE}

In this section we prove Propn. 4.5 and Propn. 4.6, completing the derivation of the LDE. We place both results under the heading of "capacity estimates" because they are proved by estimating hitting probabilities of Brownian motion. 
Lemma 4.13. Let $D \equiv \mathbb{H} \backslash \overline{\mathbb{D}}$, and let $W_{t}$ be Brownian motion started in $D$ and stopped at $\tau \equiv \tau_{D}$. If $p(z, w)$ denotes the density of $W_{\tau}$ on $\partial D$ with respect to Lebesgue measure, then

$$
p\left(z, e^{i \theta}\right)=\frac{2}{\pi} \frac{\operatorname{Im} z}{|z|^{2}} \sin \theta\left(1+O\left(|z|^{-1}\right)\right) .
$$

Consequently, if $\operatorname{rad} A \leq 1$ then

$$
\text { hcap } A=\frac{2}{\pi} \int_{0}^{\pi} \mathbb{E}_{e^{i \theta}}\left[\operatorname{Im} W_{\tau_{\mathbb{H} \backslash A}}\right] \sin \theta d \theta .
$$

Proof. Recall the Poisson integral formula for $\mathbb{H}$ given in (2.2). If $f$ is a bounded continuous function on $\partial D$ with $f=0$ outside of $\partial \mathbb{D}$, then

$$
\mathbb{E}_{z}\left[f\left(W_{\tau}\right)\right]=P_{D} f(z)=P_{\mathbb{H}}\left(f \circ \varphi^{-1}\right)(\varphi(z)),
$$

where $\varphi(z)=z+1 / z$ maps $D$ conformally onto $\mathbb{H}$. In particular $\varphi$ maps $e^{i \theta}$ to $2 \cos \theta \in[-2,2]$, so

$$
\begin{aligned}
\mathbb{E}_{z}\left[f\left(W_{\tau}\right)\right] & =\frac{1}{\pi} \int_{-2}^{2} Q_{\operatorname{Im} \varphi(z)}(t-\operatorname{Re} \varphi(z)) f \circ \phi^{-1}(t) d t \\
& =\frac{2}{\pi} \int_{0}^{\pi} Q_{\operatorname{Im} \varphi(z)}(2 \cos \theta-\operatorname{Re} \varphi(z)) f\left(e^{i \theta}\right) \sin \theta d \theta .
\end{aligned}
$$

(4.8) then follows since $\operatorname{Re} \varphi(z)=\left(1+|z|^{-2}\right) \operatorname{Re} z$ and $\operatorname{Im} \varphi(z)=\left(1-|z|^{-2}\right) \operatorname{Im} z$. Next, by Propn. 4.4 and the strong Markov property for Brownian motion, if $\operatorname{rad} A \leq 1$ and $|z|>1$ then

$$
\operatorname{Im}\left[z-g_{A}(z)\right]=\frac{2}{\pi} \frac{\operatorname{Im} z}{|z|^{2}} \int_{0}^{\pi} \mathbb{E}_{e^{i \theta}}\left[\operatorname{Im} W_{\tau_{\text {II } \backslash A}}\right] \sin \theta\left(1+O\left(|z|^{-2}\right)\right) d \theta .
$$

Taking $z=i y$ and taking $y \uparrow \infty$ gives (4.9).

Proof of Propn. 4.5. By scaling we may assume $\operatorname{rad} A=1$. For $z \in \mathbb{H} \backslash A$, let $h(z) \equiv g_{A}(z)-z-\operatorname{hcap} A / z$ and consider

$$
v(z) \equiv \operatorname{Im} h(z)=\operatorname{Im}\left[g_{A}(z)-z\right]+\operatorname{hcap} A \frac{\operatorname{Im} z}{|z|^{2}}
$$

By (4.9) and (4.10), there exists a constant $c<\infty$ such that

$$
|v(z)| \leq c \frac{\operatorname{Im} z}{|z|^{3}} \operatorname{hcap} A .
$$

On the other hand, if $\overline{B_{r}(z)} \subset \mathbb{H} \backslash A$, the Poisson integral formula (2.1) for the disc gives

$$
v(w)=\frac{1}{2 \pi} \int_{0}^{2 \pi} \operatorname{Re}\left(\frac{R+(w-z)}{R-(w-z)}\right) v\left(z+R e^{i \varphi}\right) d \varphi, \quad w \in B_{r}(z),
$$


and differentiating we find that for $z$ sufficiently far away from $A$ (say $|z| \geq 2$ ),

$$
\left|\frac{\partial v}{\partial x}(z)\right|,\left|\frac{\partial v}{\partial y}(z)\right| \leq c \frac{\mathrm{hcap} A}{|z|^{3}} .
$$

It follows by the Cauchy-Riemann equations that $\left|h^{\prime}(z)\right| \leq c$ (hcap $\left.A\right) /|z|^{3}$, and since $\lim _{r \rightarrow \infty} h\left(r e^{i \theta}\right)=0$ we can integrate from $\infty$ to bound $h\left(r e^{i \theta}\right)$ :

$$
\left|h\left(r e^{i \theta}\right)\right|=\left|\int_{y}^{\infty} h^{\prime}\left(t e^{i \theta}\right) d t\right| \leq c \frac{\mathrm{hcap} A}{r^{2}}
$$

which concludes the proof.

It remains to prove Propn. 4.6, which is done by relating the size of sets to hitting probabilities of Brownian motion. For $B$ a subset of $\overline{\mathbb{H}}$ and $A \in \mathcal{Q}$, define the capacity of $B$ relative to $A$ by

$$
\operatorname{cap}_{\mathbb{H}}(B ; A) \equiv \lim _{y \rightarrow \infty} y \mathbb{P}_{i y}\left(W\left[0, \tau_{\mathbb{H} \backslash A}\right] \cap B \neq \varnothing\right) .
$$

We call $\operatorname{cap}_{\mathbb{H}}(B) \equiv \operatorname{cap}_{\mathbb{H}}(B ; \varnothing)$ the capacity of $B$. This capacity scales as $\operatorname{cap}_{\mathbb{H}} r B=r \operatorname{cap}_{\mathbb{H}} B$, and has the following invariance property: for $B^{\prime} \subseteq \mathbb{H}$, let $g_{A}\left(B^{\prime}\right)$ denote the pre-image of $B^{\prime}$ under the continuation of $g_{A}^{-1}$ to $\overline{\mathbb{H}}$. Then:

Lemma 4.14. For $A \in \mathcal{Q}, \operatorname{cap}_{\mathbb{H}} A=\operatorname{cap}_{\mathbb{H}} \widehat{A}=\pi^{-1}$ len $\widehat{A}$ where $\widehat{A}=g_{A}(\bar{A})$ is an interval in $\mathbb{R}$.

Proof. Let $\sigma \equiv \tau_{\mathbb{H}}$. If $\widehat{A}$ is any interval in $\mathbb{R}$, the Poisson integral formula (2.2) for $\mathbb{H}$ gives

$$
y \mathbb{P}_{i y}\left(W_{\sigma} \in \widehat{A}\right)=\frac{1}{\pi} \int_{\widehat{A}} \frac{y^{2}}{(t-x)^{2}+y^{2}} d t,
$$

which tends to $\pi^{-1}$ len $\widehat{A}$ as $y \rightarrow \infty$. For $A \in \mathcal{Q}$, by the conformal invariance of Brownian motion, $\operatorname{cap}_{\mathbb{H}} \bar{A}=\lim _{y \rightarrow \infty} y \mathbb{P}_{g_{A}(i y)}\left(W_{\sigma} \in \widehat{A}\right)$. By the hydrodynamic normalization $g_{A}(i y)=u_{A}(i y)+i v_{A}(i y)=i y+O\left(y^{-1}\right)$, therefore

$$
\operatorname{cap}_{\mathbb{H}} A=\lim _{y \rightarrow \infty} y \mathbb{P}_{v_{A}(i y)}\left(W_{\sigma} \in \widehat{A}-u_{A}(i y)\right)=\lim _{y \rightarrow \infty} y \mathbb{P}_{i y}\left(W_{\sigma} \in \widehat{A}\right)=\operatorname{cap}_{\mathbb{H}} \widehat{A},
$$

which concludes the proof.

An immediate consequence is that $\operatorname{cap}_{\mathbb{H}} A \asymp \operatorname{diam} A$, so to prove Propn. 4.6 it suffices to estimate the capacity of $g_{s}(\gamma(s, t])$. To this end, here is a simple consequence of Lem. 4.14:

Corollary 4.15. Let $A \in \mathcal{Q}, B \subset \overline{\mathbb{H}}$ such that $A^{\prime} \equiv(A \cup B) \cap \mathbb{H} \in \mathcal{Q}$. Then $\operatorname{cap}_{\mathbb{H}}(B ; A)=\operatorname{cap}_{\mathbb{H}} g_{A}(B)$.

Proof. Let $\sigma \equiv \tau_{\mathbb{H}}$. By the conformal invariance of Brownian motion,

$$
\operatorname{cap}_{\mathbb{H}}(B ; A)=\lim _{y \rightarrow \infty} y \mathbb{P}_{g_{A^{\prime}}(i y)}\left(W_{\sigma} \in g_{A^{\prime}}(B)\right)=\lim _{y \rightarrow \infty} y \mathbb{P}_{i y}\left(W_{\sigma} \in g_{A^{\prime}}(B)\right),
$$


where the second identity follows from the calculation of Lem. 4.14. Since $g_{A^{\prime}}=$ $\tilde{g} \circ g_{A}$ where $\tilde{g}=g_{g_{A}\left(A^{\prime} \backslash A\right)}$, it follows similarly that

$$
\operatorname{cap}_{\mathbb{H}} g_{A}(B)=\lim _{y \rightarrow \infty} y \mathbb{P}_{\tilde{g}(i y)}\left(W_{\sigma} \in \tilde{g} \circ g_{A}(B)\right)=\lim _{y \rightarrow \infty} y \mathbb{P}_{i y}\left(W_{\sigma} \in g_{A^{\prime}}(B)\right),
$$

which proves $\operatorname{cap}_{\mathbb{H}}(B ; A)=\operatorname{cap}_{\mathbb{H}} g_{A}(B)$.

The intersection probabilities of Brownian motion can be estimated by the following very useful result:

Theorem 4.16 (Beurling estimate). Suppose $0<r_{1}<r_{2}<\infty$ and $\gamma:[0,1] \rightarrow$ $\mathbb{C}$ is a curve from $C_{r_{1}}$ to $C_{r_{2}}$. Then

$$
\begin{aligned}
& \mathbb{P}_{z}\left(W\left[0, \tau_{B_{r_{2}}}\right] \cap \gamma=\varnothing\right) \lesssim\left(r_{1} / r_{2}\right)^{1 / 2}, \quad|z| \leq r_{1}, \\
& \mathbb{P}_{z}\left(W\left[0, \tau_{\mathbb{C} \backslash \overline{B_{r_{1}}}}\right] \cap \gamma=\varnothing\right) \lesssim\left(r_{1} / r_{2}\right)^{1 / 2}, \quad|z| \geq r_{2} .
\end{aligned}
$$

For a proof see $[72, \S 3.8]$. This estimate allows us to prove the main lemma:

Proof of Propn. 4.6. Write $d \equiv \operatorname{diam} \gamma$ and $r \equiv \sup \{\operatorname{Im} z: z \in B\}$ : by scaling it suffices to show $\operatorname{cap}_{\mathbb{H}} g_{A}(B) \lesssim d^{1 / 2}$ when $r=1, d<1 / 2$ (say). By Cor. 4.15,

$$
\operatorname{cap}_{\mathbb{H}} g_{A}(B)=\operatorname{cap}_{\mathbb{H}}(B ; A)=\lim _{y \rightarrow \infty} y \mathbb{P}_{i y}(W[0, \tau] \cap B \neq \varnothing), \quad \tau \equiv \tau_{\mathbb{H} \backslash A} .
$$

Fix $z_{0} \in B$. For large $y, \mathbb{P}_{i y}\left(W[0, \tau] \cap B_{1}\left(z_{0}\right) \neq \varnothing\right) \lesssim c / y$ (e.g. by Lem. 4.14). Once the Brownian motion enters $B_{1}\left(z_{0}\right)$, the probability that it intersects $B$ before leaving $\mathbb{H} \backslash A$ is bounded above by the probability that it enters $B_{2 d}\left(z_{0}\right)$ before leaving $\mathbb{H} \backslash A$. This is $\lesssim d^{1 / 2}$ by Thm. 4.16 so the result follows.

The results of this section concerned the hypothetical scaling limits of random curves arising in discrete physical models such as LERW and percolation. We return in the next two sections to the proof of Thm. 3.12.

\section{Scaling limits}

In this section we present a result of Aizenman and Burchard [5] which guarantees the existence of subsequential weak limits for the percolation exploration paths $\gamma^{\delta}$ in discrete domains $D_{2}^{\delta}$ converging to a limit $D_{2}$. The next section presents the work of Smirnov and Camia and Newman which pins down the limit to be $\mathrm{SLE}_{6}$. Throughout this section we assume that $D$ is a bounded domain in $\mathbb{R}^{d}$; without loss $\operatorname{diam} D \leq 1$.

\subsection{Systems of random curves}

Recall Defn. 3.3 of the space of curves $\mathcal{C}$ with the uniform metric $d_{\mathcal{U}}$; this is a complete separable metric space. Given a bounded domain $D$, let $\mathcal{C}_{D}$ denote the subspace of $\mathcal{C}$ of curves traveling in the closure of $D$. To formalize the notion of a "discrete curve," let $\mathcal{C}_{D}^{\delta}$ denote the subspace of $\mathcal{C}_{D}$ of polygonal curves of step size $\delta$. 
Definition 5.1. A curve configuration is a closed subset of $\mathcal{C}_{D}$; it is a $\delta$ curve configuration if it is contained entirely in $\mathcal{C}_{D}^{\delta}$. Denote the space of curve configurations by $\Omega_{D}$ and the space of $\delta$-curve configurations by $\Omega_{D}^{\delta}$.

A metric on $\Omega_{D}$ is given by the Hausdorff metric induced by the metric $d_{\mathcal{U}}$ on $\mathcal{C}_{D}$ : for $F, F^{\prime} \in \Omega_{D}$, the Hausdorff distance $d_{\mathcal{H}}\left(F, F^{\prime}\right)$ between them is the smallest $\epsilon>0$ such that each is contained in the $\epsilon$-neighborhood of the other (with respect to $d_{\mathcal{U}}$ ). With this metric, the completeness and separability of $\mathcal{C}_{D}$ is passed on to $\Omega_{D}$.

In this section we study (random) systems of configurations, collections $F=\left(F^{\delta}\right)_{0<\delta \leq \delta_{0}}$ of random variables where the law $\mathbb{P}^{\delta}$ of $F^{\delta}$ is a probability measure on $\Omega_{D}$ (with the Borel $\sigma$-algebra) with support contained in $\Omega_{D}^{\delta}$.

The aim is to specify a regularity condition, verifiable in the percolation setting, which implies precompactness of $\left\{\mathbb{P}^{\delta}\right\}$ in the weak topology: this means that along any sequence $\delta \rightarrow 0$ we can extract a subsequence $\delta_{n}$ with $\mathbb{P}^{\delta_{n}}$ converging weakly to a limiting measure on $\Omega_{D}$. In view of Thm. 3.7, it is natural to seek a regularity condition formulated in terms of crossings of annuli. Let $A(x ; r, R) \equiv B_{R}(x) \backslash \overline{B_{r}(x)}$.

Definition 5.2. Let $k \in \mathbb{N}, 0<r<1, \eta>0, x \in \bar{D}$. A curve $\gamma \in \mathcal{C}_{D}$ has a $k$-fold crossing of power $\eta$ and scale $r$ (for short, a ( $k, \eta, r)$-crossing) at $x$ if the annulus $A\left(x ; r^{1+\eta}, r\right)$ is traversed by $k$ separate segments of $\gamma$.

Let $E(k, \eta, r)$ denote the set of $F \in \Omega_{D}$ exhibiting a $(k, \eta, r)$-crossing (i.e., such that some $\gamma \in F$ has a $(k, \eta, r)$-crossing).

Definition 5.3. Let $\left(F^{\delta}\right)_{0<\delta \leq \delta_{0}}$ be a system of configurations specified by laws $\left(\mathbb{P}^{\delta}\right)_{0<\delta \leq \delta_{0}}$. We say the system has uniform crossing exponents if for all $k \in$ $\mathbb{N}$ there exist constants $c_{k}, \lambda_{k}<\infty$ with $\lambda_{k} \rightarrow \infty$ such that for any $0<r<1$,

$$
\mathbb{P}^{\delta}[E(k, \eta, r)] \leq c_{k} r^{-\lambda_{k} \eta} .
$$

Theorem 5.4 (Aizenman, Burchard [5]). Let $\left(F^{\delta}\right)_{0<\delta \leq \delta_{0}}$ be a system of configurations specified by laws $\left(\mathbb{P}^{\delta}\right)_{0<\delta \leq \delta_{0}}$. If the system has uniform crossing exponents, then the family $\left\{\mathbb{P}^{\delta}\right\}$ is precompact.

Here is the application to our problem of interest: let $F^{\delta}$ be the percolation configuration on the discrete domain $D_{2}^{\delta}$, regarded as the collection of all interface curves not including the domain boundary.

Corollary 5.5. Let $D_{2}^{\delta}$ be discrete domains converging uniformly to the twomarked domain $D_{2}$ (with the spherical metric). If $\mathbb{P}^{\delta}$ is the law of the percolation configuration $F^{\delta}$ then the family $\left\{\mathbb{P}^{\delta}\right\}$ is precompact.

Proof. Thm. 3.7 implies the existence of a 1-arm crossing exponent $\lambda_{1}$, and the BK inequality implies the $k$-arm crossing exponent $\lambda_{k}=k \lambda_{1}$, so the result follows from Thm. 5.4.

Each $F^{\delta}$ has a distinguished curve $\gamma^{\delta}$ (the exploration path), and by applying Thm. 5.4 again we can extract a further subsequence along which $\gamma^{\delta}$ converges 
weakly to a limit curve $\gamma$. Consequently, to prove Thm. 3.12 it remains to identify any such limit $\gamma$ as an SLE $_{6}$ curve. This will be done in the next section using the characterization of Propn. 4.12.

We turn now to the proof of Thm. 5.4. By Prohorov's theorem (see e.g. [18]) it suffices to show that the family is tight, i.e. that for all $\epsilon>0$ there exists $\mathcal{K} \subset \Omega_{D}$ compact such that $\mathbb{P}^{\delta}(\mathcal{K}) \geq 1-\epsilon$ for all $\delta$. Compactness in $\Omega_{D}$ can be characterized as follows:

Lemma 5.6. If $\mathcal{K}$ is a closed subset of $\Omega_{D}$ such that the union of all $K \in \mathcal{K}$ is contained in some $K_{0} \subset \mathcal{C}_{D}$ compact, then $\mathcal{K}$ is compact.

Proof. By elementary topology, if $(S, d)$ is a compact metric space then the space $\mathcal{H}(S)$ of closed subsets of $S$ taken with the Hausdorff metric $d_{\mathcal{H}}$ is also compact (see e.g. [88, p. 280]). Under the hypothesis, $\mathcal{K}$ is a closed subset of the compact space $\left(\mathcal{H}\left(K_{0}\right), d_{\mathcal{H}}\right)$ and hence is compact.

Compactness in $\mathcal{C}_{D}$ is in turn characterized by the Arzelà-Ascoli theorem, so to prove tightness it suffices to prove an equicontinuity bound which holds with probability $\geq 1-\epsilon$ under each $\mathbb{P}^{\delta}$. Towards this end, the next section relates Hölder continuity to annuli crossings.

\subsection{Hölder, tortuosity, and dimension bounds}

The optimal Hölder exponent of a curve $\gamma$, denoted $\alpha(\gamma)$, is the supremum of all $\alpha>0$ such that $\gamma$ admits a parametrization which is Hölder continuous with exponent $\alpha$-i.e., such that

$$
|\gamma(s)-\gamma(t)| \leq c_{\alpha}|s-t|^{\alpha} \quad \forall s, t \in[0,1]
$$

for some constant $c_{\alpha}<\infty$. Inverting this relation gives the equivalent condition

$$
|s-t| \geq c_{\alpha}^{\prime}|\gamma(s)-\gamma(t)|^{1 / \alpha} \quad \forall s, t \in[0,1] .
$$

That is, if two points on the curve are a certain distance apart, Hölder continuity puts a lower bound on their time difference. Motivated by this observation, let $M_{r}(\gamma)$ denote the minimal $n$ such that $\gamma$ can be partitioned into $n$ segments of diameter $\leq r$; this is a measure of the curve's "tortuosity." The tortuosity exponent of $\gamma$ is defined as

$$
\tau(\gamma) \equiv \inf \left\{s>0: \lim _{r \downarrow 0} r^{s} M_{r}(\gamma)=0\right\}
$$

Proposition 5.7. For $\gamma \in \mathcal{C}_{D}, \alpha(\gamma)=1 / \tau(\gamma)$.

Proof. If $\psi$ is an increasing homeomorphism of $[0,1]$ such that $\psi\left(\left|\gamma\left(t_{1}\right)-\gamma\left(t_{2}\right)\right|\right) \leq$ $\left|t_{1}-t_{2}\right|$ whenever $\left|\gamma\left(t_{1}\right)-\gamma\left(t_{2}\right)\right| \leq 1$, then certainly $M_{r}(\gamma) \leq\lceil 1 / \psi(r)\rceil$ for all $0<r \leq 1$, and it follows that $\tau(\gamma) \leq \alpha(\gamma)^{-1}$. 
Suppose conversely that $M_{r}(\gamma) \leq 1 / \psi(r)$ for $0<r \leq 1$. For a curve $\eta \in \mathcal{C}_{D}$, define

$$
\tilde{t}(\gamma) \equiv \sum_{n \geq 0} \frac{\psi\left(r_{n}\right) M_{r_{n}}(\gamma)}{(n+1)^{2}}, \quad r_{n} \equiv 2^{-n}
$$

Take an auxiliary parametrization $\gamma_{0}$ for $\gamma$, and consider

$$
t(u) \equiv \frac{\tilde{t}\left(\gamma_{u}\right)}{\tilde{t}(\gamma)}, \quad 0 \leq u \leq 1
$$

(Notice $\tilde{t}(\gamma) \leq 2$ by the assumed bound on $M_{r}(\gamma)$ ). Then $t:[0,1] \rightarrow[0,1]$ is a strictly increasing right-continuous function. Its generalized inverse $u$ is continuous, so we may reparametrize $\gamma(t)=\gamma_{0}(u(t))$. Let $r=|\gamma(s)-\gamma(t)|$ for $s<t$ : then $M_{r_{n}}\left(\gamma_{t}\right)-M_{r_{n}}\left(\gamma_{s}\right) \geq 1$ for all $r_{n} \leq r$, i.e. for all $n \geq \log _{2}(1 / r)$, so

$$
t-s \geq \frac{\psi(r)}{2\left(\log _{2}(1 / r)+1\right)^{2}} .
$$

This implies $\alpha(\gamma) \geq \tau(\gamma)^{-1}$ which concludes the proof.

Tortuosity in general is difficult to compute or estimate, but under regularity conditions $M_{r}(\gamma)$ can be bounded by quantities which depend only on the curve's trace, the set $\{\gamma(t): t \in[0,1]\}$. For example, let $N_{r}(\gamma)$ denote the minimal $n$ such that the trace of $\gamma$ can be covered by $n$ sets of diameter $r$. The upper box dimension or Minkowski dimension of the curve is

$$
\overline{\operatorname{dim}}_{B}(\gamma) \equiv \inf \left\{s>0: \lim _{r \downarrow 0} r^{s} N_{r}(\gamma)=0\right\} .
$$

Trivially $N_{r}(\gamma) \leq M_{r}(\gamma)$ so $\overline{\operatorname{dim}}_{B} \leq \tau$. Further it is easy to see that if $r^{\prime}<r$ then

$$
N_{r^{\prime}}(\gamma) \leq N_{r}(\gamma)\left\lceil r / r^{\prime}\right\rceil^{d},
$$

so $\overline{\operatorname{dim}}_{B} \leq d$ while $\tau$ is unbounded.

Recall Defn. 5.2 of a $(k, \eta, r)$-crossing. Let us say that a curve $\gamma$ has the tempered crossing property if for all $0<\eta<\eta_{0}$ there exist $k, r_{0}$ (both depending on $\eta$ ) such that $\gamma$ exhibits no $(k, \eta, r)$-crossings.

Proposition 5.8. If $\gamma \in \mathcal{C}_{D}$ exhibits no $(k, \eta, r)$-crossings then

$$
M_{2 r}(\gamma) \leq k N_{r^{1+\eta}}(\gamma)
$$

Consequently, if $\gamma$ has the tempered crossing property then $\overline{\operatorname{dim}}_{B}(\gamma)=\tau(\gamma)$.

Proof. We already noted $\overline{\operatorname{dim}}_{B} \leq \tau$ so it remains to prove the reverse inequality. Fix $\eta>0$ and let $k, r$ be as given by the tempered crossing property. Partition the curve as follows: set $t_{0}=0$, and for $j>0$ let

$$
t_{j}=\inf \left\{t>t_{j-1}:\left|\gamma(t)-\gamma\left(t_{j-1}\right)\right|\right\} \geq 2 r,
$$


provided this time is well-defined. If $\gamma$ does not leave $B_{2 r}\left(\gamma\left(t_{j-1}\right)\right)$, terminate by setting $t_{j}=1$.

The number of segments is an upper bound for $M_{2 r}(\gamma)$. Consider any covering of $\gamma$ by balls of radius $r^{1+\eta}$ : since $\gamma$ has no $(k, \eta, r)$-crossings and $\left|\gamma\left(t_{j}\right)-\gamma\left(t_{j-1}\right)\right| \geq$ $2 r$, each ball can contain at most $k$ of the points $\gamma\left(t_{j}\right)$. This implies $(5.2)$, and so $\tau(\gamma) \leq(1+\eta) \overline{\operatorname{dim}}_{B}(\gamma)$. The result for a curve with the tempered crossing property follows by taking $\eta \downarrow 0$.

\subsection{Proof of tightness}

We now prove Thm. 5.4. Recall $\left(F^{\delta}\right)_{0<\delta \leq \delta_{0}}$ is a system of configurations specified by laws $\left(\mathbb{P}^{\delta}\right)_{0<\delta \leq \delta_{0}}$.

Lemma 5.9. Under the hypotheses of Thm. 5.4, for all $\epsilon, \eta>0$ there exist $k, r_{0}$ such that

$$
\mathbb{P}^{\delta}\left[F^{\delta} \in E(k, \eta, r) \text { for some } r \leq r_{0}\right]<\epsilon
$$

for all $0<\delta \leq \delta_{0}$.

Proof. Suppose $F^{\delta}$ has a $(k, \eta, r)$-crossing at $x_{0} \in D$ for $r \leq 4^{-1 / \eta}$. If $x$ is any point in $B_{r^{1+\eta} / 2}\left(x_{0}\right)$, then $F^{\delta}$ exhibits a $k$-fold crossing of $A\left(x ; 2 r^{1+\eta}, r / 2\right)$, hence also of $A\left(x ; 2 r_{n}^{1+\eta}, r_{n+1} / 2\right)$ where $r_{n+1}<r \leq r_{n}\left(r_{n} \equiv 2^{-n}\right)$. Applying the crossing exponent hypothesis for shells $A\left(x ; 2 r_{n}^{1+\eta}, r_{n+1} / 2\right)$ centered at an $r_{n}^{1+\eta} / 2$-net of points $x \in D$ gives

$$
\mathbb{P}^{\delta}\left[F^{\delta} \in E(k, \eta, r) \text { for some } r_{n+1}<r \leq r_{n}\right] \leq c_{k}^{\prime} \frac{r^{\eta \lambda_{k}}}{r^{(1+\eta) d}} .
$$

Choose $k$ large enough (depending on $\eta$ ) so that the exponent on $r$ is positive, and sum over scales $r_{n} \leq r_{0}$ to conclude the result.

Define the random variables

$$
N^{\delta}(r, R) \equiv \sup \left\{N_{r}(\gamma): \gamma \in F^{\delta}, \operatorname{diam} \gamma \geq R\right\}
$$

For each $\delta, N^{\delta}(r, R)$ is nonincreasing in $r, R$.

Lemma 5.10. Under the hypotheses of Thm. 5.4, the random variables

$$
X^{\delta} \equiv \sup _{R} \sup _{r \leq R} \frac{N^{\delta}(r, R) r^{d}}{(r / R)^{\lambda}} \ell(r)
$$

where $\lambda \equiv \lambda_{1}$ and $\ell$ is a polylogarithmic factor, are stochastically bounded.

Proof. Since $D$ is a bounded domain, it suffices to obtain a bound over all scales $0<r \leq R \leq 1$. For $r>0$ let $\Pi_{r}$ denote the standard grid partition of $\mathbb{R}^{d}$ into rectangles of diameter $r$. For $r \leq R$ let $\widetilde{N}^{\delta}(r, R)$ denote the number of sets $B \in \Pi_{r}$ meeting some curve in $F^{\delta}$ of diameter $\geq R$; clearly $N^{\delta}(r, R) \leq \widetilde{N}^{\delta}(r, R)$.

For each $B \in \Pi_{r}$ let $x_{B}$ be a point such that $B \subseteq B_{r / 2}\left(x_{B}\right)$. If $B \in \Pi_{\ell}$ meets a curve of diameter $\geq R$, we must have a crossing of the spherical shell 
$A\left(x_{B} ; r / 2, R / 2\right)$, which by hypothesis occurs with probability $\leq c_{1}(r / R)^{\lambda}$. Summing over the $\asymp r^{-d}$ sets in $\Pi_{r}$ gives

$$
\mathbb{E}_{\delta}\left[\widetilde{N}^{\delta}(r, R)\right] \leq c r^{-d}(r / R)^{\lambda} .
$$

To obtain a bound over all scales, let

$$
U^{\delta} \equiv \sum_{m \geq 0} \frac{1}{(m+1)^{2}} \sum_{n \geq m} \frac{1}{(n+1)^{2}} \frac{\widetilde{N}_{r_{n}, R_{m}}^{\delta}}{\mathbb{E}\left[\widetilde{N}_{r_{n}, R_{m}}^{\delta}\right]}, \quad r_{n} \equiv 2^{-n}, R_{m} \equiv 2^{-m} .
$$

$\mathbb{E}_{\delta}\left[U^{\delta}\right]=1$ so the $U^{\delta}$ are stochastically bounded by Markov's inequality. If $R_{m} \leq R<R_{m-1}$ and $r_{n} \leq r<r_{n-1}$ then

$$
\begin{aligned}
& \widetilde{N}^{\delta}(r, R) \leq \widetilde{N}^{\delta}\left(r_{n}, R_{m}\right) \leq U^{\delta}(n+1)^{4} \mathbb{E}\left[\widetilde{N}^{\delta}\left(r_{n}, R_{m}\right)\right] \\
& \leq U^{\delta}\left[\log _{2}(1 / r)+2\right]^{4} r^{-d}(r / R)^{\lambda} .
\end{aligned}
$$

This bound holds simultaneously for all $r \leq R$ so the result follows.

Proof of Thm. 5.4. We can always decrease the $\lambda_{k}$, so assume without loss that $\lambda<d$. Let $\epsilon, \eta>0$, and let $k, r_{0}$ be as given by Lem. 5.9, so that the bound (5.2) holds with $\mathbb{P}^{\delta}$-probability $>1-\epsilon$ for all $0<\delta \leq \delta_{0}$. Then by Lem. 5.10 there exists $C<\infty$ such that

$$
M_{r}(\gamma) \leq \frac{C}{(\operatorname{diam} \gamma)^{\lambda}} \frac{\ell\left(r^{1+\eta}\right)}{r^{(1+\eta)(d-\lambda)}} \quad \forall r \leq \operatorname{diam} \gamma, \forall \gamma \in F^{\delta}
$$

holds with $\mathbb{P}^{\delta}$-probability $\geq 1-2 \epsilon$ for all $0<\delta \leq \delta_{0}$. Inverting this (as in the proof of Propn. 5.7) gives the Hölder bound

$$
|\gamma(s)-\gamma(t)| \leq C \ell(r) g(\operatorname{diam} \gamma)|s-t|^{1 /[(1+\eta)(d-\lambda)]},
$$

where $\ell$ is a (different) polylogarithmic factor and $g(R) \equiv R^{-\lambda /[(1+\eta)(d-\lambda)]}$. Interpolating between this and the trivial bound $|\gamma(s)-\gamma(t)| \leq \operatorname{diam} \gamma$ gives

$$
|\gamma(s)-\gamma(t)| \leq C \ell(r)|s-t|^{1 /[(1+\eta)(d-\lambda)+\lambda]} .
$$

Thus we have found a Hölder continuity bound holding with $\mathbb{P}^{\delta}$-probability $\geq$ $1-2 \epsilon$ for all $0<\delta \leq \delta_{0}$, and tightness follows by the Arzelà-Ascoli theorem.

Remark 5.11. Notice that (5.3), Propn. 5.7, and the relation $\overline{\operatorname{dim}}_{B} \leq \tau$ imply that the limiting curves have Minkowski dimension strictly less than $d$.

\section{Limit of the exploration path}

In this final section we present a proof of Thm. 3.12. Our exposition is based on the work of Binder, Chayes, and Lei [19, 20] and of Camia and Newman [31].

By the results of $\S 5$, the set of laws $\mathbb{P}^{\delta}$ of the percolation configurations (regarded as curve configurations) is precompact: from any sequence $\delta \downarrow 0$ we 
can extract a further sequence along which $\mathbb{P}^{\delta}$ converges weakly, to a limit which depends a priori on the particular subsequence. It remains therefore to uniquely identify the weak limits, which will be done in $\$ 6.2$ using the characterization of Propn. 4.12. Before doing this, however, we need to address some issues concerning discrete approximation for $k$-marked domains which were ignored in $\S 3$ (see Rmk. 3.6): this is the topic of $\S 6.1$.

Remark 6.1. Whenever we have a precompact family of probability measures on a separable space we will assume that we work within a weakly convergent subsequence, and further, by the Skorohod coupling theorem (see Rmk. 3.13), that this sequence has an a.s. convergent coupling.

Specifically, by Cor. 5.5 and the subsequent comments, we may assume that along our subsequence $F^{\delta} \rightarrow F$ with respect to the Hausdorff metric on $\Omega_{D}$, and that $\gamma^{\delta} \rightarrow \gamma$ uniformly.

\subsection{Admissible domains and discrete approximation}

Recall the notation for $k$-marked domains introduced in Defns. 3.1 and 3.2. Clearly, even if $D_{2}$ is restricted to be a Jordan domain, to prove this result one needs to consider more generally the "slit domains" $\left(D \backslash K_{t} ; \gamma(t), b\right)$, where $K_{t}$ is the filling of $\gamma[0, t]$ (see $\S 4.2$ ). A useful notion here is that of prime end, first introduced by Carathéodory [32]. We omit a formal definition (see [45, 90]), but roughly speaking a prime end of $D$ is a "conformal boundary point" - it may not be a boundary point itself, but it "corresponds" to a boundary point of $\mathbb{D}$ under conformal mappings $\mathbb{D} \rightarrow D$. For example, if $D=\mathbb{D} \backslash[0,1]$, the point $1 / 2$ "splits" into two distinct prime ends: any conformal map $\mathbb{D} \rightarrow D$ has a continuous extension to the unit circle by Carathéodory's theorem, and two distinct points will map to $1 / 2$.

Definition 6.2. A (generalized) $k$-marked domain means a domain $D$ whose boundary $\partial D$ is a continuous closed curve $\eta:[0,1] \rightarrow \mathbb{C}$, with marked prime ends $P_{i}=\eta\left(t_{i}\right)$ for $0=t_{1} \leq \cdots \leq t_{k} \leq 1$. The domain is admissible if $\eta$ is simple on each $\left[t_{i}, t_{i+1}\right]$.

A rather more subtle point is that we need in addition a more general notion of discrete approximation than that of Defn. 3.4. A natural form of convergence for complex domains is Carathéodory convergence: for complex domains $D_{n}, D$ it is said that $D_{n}$ converges to $D$ in the Carathéodory sense if

(i) $z \in D$ implies $z \in \liminf D_{n}$, and

(ii) $z_{n} \notin D_{n}, z_{n} \rightarrow z$ implies $z \notin D$.

Note that a single sequence can have multiple Carathéodory limits, for example the doubly slit domain $\mathbb{C} \backslash((-\infty, 1 / n] \cup[1 / n, \infty))$ converges to both $\mathbb{H}$ and $-\mathbb{H}$.

Carathéodory kernel theorem. Let $D_{n}, D$ complex domains. There exist conformal maps $f_{n}: \mathbb{H} \rightarrow D_{n}, f: \mathbb{H} \rightarrow D$ with $f_{n} \rightarrow f$ locally uniformly in $\mathbb{H}$ if and only if $D_{n} \rightarrow D$ in the Carathéodory sense. 
However, as noted in [20], Carathéodory convergence is insufficient for the verification of Cardy's formula because of boundary issues. This motivates the following (stronger) definition of domain convergence:

Definition 6.3. Let $D_{k}^{\delta}, D_{k}$ be admissible $k$-marked domains. We say $D_{k}^{\delta}$ converges conformally to $D_{k}$, denoted $D_{k}^{\delta} \rightsquigarrow D_{k}$, if each $A_{j}^{\delta}$ converges uniformly to a curve $A_{j}^{\star}$ traveling from $P_{j}$ to $P_{j+1}$ such that

(i) $A_{j} \subseteq A_{j}^{\star} \subset \mathbb{C} \backslash\left(D \cup\left(\bigcup_{\ell \neq j} A_{\ell}\right)\right)$ (as sets), and

(ii) both $A_{j}^{\star}$ and its reverse are self-avoiding. ${ }^{9}$

Conformal convergence is weaker than the uniform convergence of Defn. 3.4 because we only require $A_{j}^{\star}$ to agree with $A_{j}$ from the "perspective" of the domain interior; $A_{j}^{\star}$ is allowed to make excursions away from $\partial D$. We leave the reader to verify that the proof of Thm. 3.5 also implies the following generalization:

Theorem 6.4. Let $D_{4}^{\delta}$ be discrete four-marked domains converging conformally to the bounded admissible domain $D_{4}$. Then

$$
\Phi\left(D_{4}^{\delta}\right) \rightarrow \Phi\left(D_{4}\right)
$$

where $\Phi\left(D_{4}\right)$ denotes the evaluation of Cardy's formula for $D_{4} \cdot{ }^{10}$

\subsection{Convergence of slit domains}

We turn finally to the verification of the conditions of Propn. 4.12, which will be done in an inductive manner as follows: let $D_{2}^{\delta}$ be discrete domains converging conformally to the bounded admissible domain $D_{2}$ (again, here $D$ is thought of as the original domain minus the filling up to some time $t$ ). Let $J$ be a crosscut of $D_{2}$ joining $c \in \overline{a b}$ to $d \in \overline{b a}$, and let $K \equiv K\left(D_{2}, J\right)$. Take conformal maps $f^{\delta}: \mathbb{H}_{2} \rightarrow D_{2}^{\delta}, f: \mathbb{H}_{2} \rightarrow D_{2}$ with $f^{\delta} \rightarrow f$ locally uniformly on $\mathbb{H}_{2}$ as given by the Carathéodory kernel theorem, and let $J^{\delta} \equiv f^{\delta} \circ f^{-1}(J)$. Consider running the discrete curve $\gamma^{\delta}$ until the first time $\tau^{\delta}$ that it reaches a hexagonal vertex on the other side of $J^{\delta}$ from the initial point. The discrete filling $K^{\delta} \equiv K^{\delta}\left(D_{2}^{\delta}, J^{\delta}\right)$ is the smallest simply connected closed set containing the union of all hexagons explored up to time $\tau^{\delta}$.

By Rmk. 6.1, we always work along a subsequence with $F^{\delta} \rightarrow F$ in $\Omega_{D}$ and $\gamma^{\delta} \rightarrow \gamma$ uniformly. In fact, we assume (passing to a further subsequence as needed) that the discrete filling boundaries $\partial K^{\delta}$ converge uniformly to a curve $\eta$. Note that $\eta$ need not agree with $\partial K$ - in fact, from our definition of conformal convergence, it need not even lie entirely in $D$ ! The following is the main content of the inductive step:

\footnotetext{
${ }^{9}$ Formally, we mean that there exists a domain $D \subset D^{\prime} \subset \mathbb{C} \backslash \bigcup_{\ell \neq j} A_{\ell}$ such that both $A_{j}^{\star}$ and its reverse travel chordally in $D^{\prime}$, and are self-avoiding in the sense of Defn. 4.8.

${ }^{10}$ Note that Cardy's formula still makes sense because we marked prime ends on the boundary of $D_{4}$.
} 
Proposition 6.5. In the setting described above, almost surely $\gamma, \eta$ are selfavoiding, $\left(D^{\delta} \backslash K^{\delta} ; \gamma\left(\tau^{\delta}\right), b^{\delta}\right) \rightsquigarrow(D \backslash K ; \gamma(\tau), b)$, and the law of $K$ is given by Cardy's formula.

As a first step, we show that the stopping rules determined by $J$ and $J^{\delta}$ are consistent:

Lemma 6.6. In the setting of Propn. 6.5, parametrize $\gamma$ by hcap and parametrize $\gamma^{\delta}$ so that $\sup _{t}\left|\gamma^{\delta}(t)-\gamma(t)\right| \rightarrow 0$. Then $\tau^{\delta} \rightarrow \tau$.

Proof. If $\tau^{\delta}$ fails to converge to $\tau$ as $\delta \downarrow 0$ this means that the curves $\gamma^{\delta}$ approach arbitrarily closely a point $z_{0} \in J^{\delta}$ without crossing, then travel a constant-order distance away (so that capacity increases) before eventually crossing $J^{\delta}$. This implies that for some $\epsilon>0$ and for all $\epsilon^{\prime}<\epsilon$, if we condition on the first time that $\gamma^{\delta}$ comes within distance $\epsilon^{\prime}$ of $J^{\delta}$ and let $z_{0}^{\delta}$ denote the nearest point on $J^{\delta}$, the curve will exit the annulus $A\left(z_{0}^{\delta} ; \epsilon^{\prime}, \epsilon\right)$ without crossing $J^{\delta}$. For fixed $\epsilon$ this probability decreases to zero as $\epsilon^{\prime} \downarrow 0$ (e.g. by Thm. 3.7), and taking $\epsilon \downarrow 0$ proves $\tau^{\delta} \rightarrow \tau$.

We now show that curves in the (subsequential) limiting percolation configuration are almost surely self-avoiding. The general method for proving such results (see e.g. [6]) is via a priori estimates on crossing events. By a nonmonochromatic $k$-arm crossing we mean $k$ disjoint crossings not all of the same color. Here is the estimate we will use to control the behavior of $\gamma$ in the domain interior:

Lemma 6.7 (Kesten, Sidoravicius, Zhang [67, Lem. 5]). The $\mathbb{P}^{\delta}$-probability of a non-monochromatic five-arm crossing of $A(z ; r, R)$ is $\asymp(r / R)^{2}$ for all $R / r, \delta / r \geq c$. Consequently the $\mathbb{P}^{\delta}$-probability of a non-monochromatic six-arm crossing of $A(z ; r, R)$ is $\lesssim(r / R)^{2+\lambda}$, where $\lambda$ is as in Thm. 3.7.

For the proof of the five-arm exponent see [67]; the six-arm exponent follows directly from Thm. 3.7 and the BK inequality. Controlling the behavior of $\gamma$ at the domain boundary is more subtle, and the following is the estimate we will require:

Lemma 6.8 (Lawler, Schramm, Werner [75, Appx. A]). Let $\Gamma$ be a fixed smooth closed contour inside $D$, and let $z \in \partial D$. Then there exists a constant $c \equiv c(\Gamma)$ such that the $\mathbb{P}^{\delta}$-probability of a non-monochromatic three-arm crossing from $B_{r}(z)$ to $\Gamma$ within $D$ is $\leq c r^{2}$.

This is not precisely the estimate which is proved in [75] but we leave the reader to check that it follows from a slight modification of their argument. We turn now to the proof of Propn. 6.5.

Proof of Propn. 6.5. Each time $\gamma$ intersects a previous part of its path or the boundary of $D$, a region is "sealed off" (disconnected from $b$ ), and to show that $\gamma$ is self-avoiding we must show that it does not dive into sealed-off regions.

(1) No triple points in interior. If $\gamma$ enters a sealed-off region in the interior of the domain, then $\gamma$ must have a triple visit at the entry point. This implies 


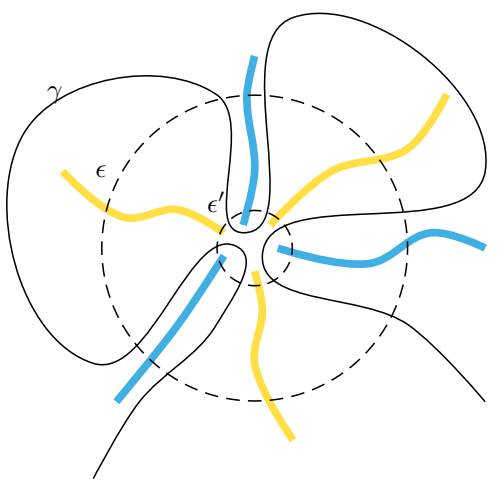

(a) Triple point without dive

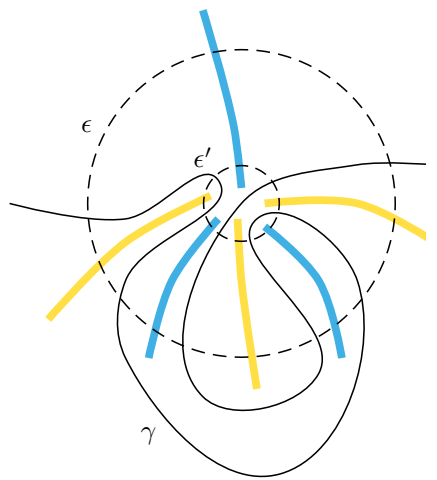

(b) Triple point with dive

Fig 10: Interior triple point and six-arm event

that for some $\epsilon>0$ and for all $\epsilon^{\prime}<\epsilon, \gamma^{\delta}$ has a six-fold crossing of some annulus $A\left(z ; \epsilon^{\prime}, \epsilon\right), z \in D$, for sufficiently small $\delta$. Fig. 10 shows two topologically distinct ways in which this can occur: although the only situation which concerns us is that of Fig. 10b, we will eliminate both possibilities. Since $\gamma^{\delta}$ has blue to the left and yellow to the right, in all cases the sixfold crossing implies a non-monochromatic six-arm crossing of $A\left(z ; \epsilon^{\prime}, \epsilon\right)$. Moreover the annuli can be taken to lie on a square grid of side length $\asymp \epsilon^{\prime}$, so summing over $\lesssim 1 /\left(\epsilon^{\prime}\right)^{2}$ annuli and applying Lem. 6.7 we find that the probability of such an event is $\lesssim\left(\epsilon^{\prime}\right)^{\lambda} / \epsilon^{2+\lambda}$. Taking first $\epsilon^{\prime} \downarrow 0$ and then $\epsilon^{\prime} \downarrow 0$ shows that there are no interior triple points.

(2) No double points on boundary. If $\gamma$ enters a sealed-off region at the domain boundary, then $\gamma$ must have a double visit at the entry point, and we claim this does not occur. Let $\varphi$ be a conformal map $\mathbb{D} \rightarrow D$ and let $\Gamma_{\epsilon} \equiv \varphi\left(C_{1-\epsilon}\right)$; note that $\operatorname{dist}\left(\Gamma_{\epsilon}, \partial D\right) \downarrow 0$ as $\epsilon \downarrow 0$ by a simple compactness argument. Thus, arguing similarly as above we see that a boundary double point implies that for some $\epsilon>0$ and all $\epsilon^{\prime}<\epsilon$, there is a non-monochromatic threearm crossing from an $\epsilon^{\prime}$-neighborhood of $\partial D$ to $\Gamma_{\epsilon}$ for sufficiently small $\delta$. But $\partial D$ has Minkowski dimension strictly less than two (Rmk. 5.11), so the claim follows by partitioning $\partial D$ into segments of diameter $\leq \epsilon^{\prime}$ and applying Lem. 6.8 with $\Gamma=\Gamma_{\epsilon}$.

(3) No retracing. We claim $\gamma$ does not trace any non-trivial segment of the boundary or of its past hull. Retracing implies lengthwise crossings as $\delta \downarrow 0$ of four-marked domains which are conformally equivalent to $\epsilon^{\prime} \times \epsilon$ rectangles for fixed $\epsilon$ and all $\epsilon^{\prime}<\epsilon$. The probability of such a crossing is $\lesssim \alpha^{\epsilon / \epsilon^{\prime}}$ for some constant $\alpha<1$. Summing over $\lesssim 1 /\left(\epsilon^{\prime}\right)^{2}$ such domains (covering $\gamma$ and $\partial D$ ) gives the result.

The entire argument above applies equally to $\eta$ so we find that both $\gamma, \eta$ are self-avoiding. Combining this with the result of Lem. 6.6 gives that $\left(D^{\delta} \backslash\right.$ $\left.K^{\delta} ; \gamma\left(\tau^{\delta}\right), b^{\delta}\right) \rightsquigarrow(D \backslash K ; \gamma(\tau), b)$, and the law of $K$ is determined by Cardy's formula by Thm. 6.4 . 
We now conclude the proof of the main theorem:

Proof of Thm. 3.12. By Propn. 6.5 the limit $\gamma$ is self-avoiding, so it remains to show that the $\epsilon$-filling sequence satisfies the characterization of Propn. 4.12.

Let $f: \mathbb{H}_{2} \rightarrow D_{2}, f^{\delta}: \mathbb{H}_{2} \rightarrow D_{2}^{\delta}$ be as above. Let $J_{1}=f\left(C_{\epsilon}^{+}\right)$and $J_{1}^{\delta}=$ $f^{\delta} \circ f^{-1}\left(J_{1}\right)$. Let $x_{1}^{\delta}$ denote the tip of $K_{1}^{\delta}$ (the point where $\gamma^{\delta} \operatorname{crosses} J^{\delta}$ ), and $x_{1}$ the tip of $K_{1}$ (its point of intersection with $J$ ), and define

$$
D_{2}^{\delta}(1) \equiv\left(D_{2}^{\delta} \backslash K_{1}^{\delta} ; x_{1}^{\delta}, b^{\delta}\right), \quad D_{2}(1) \equiv\left(D_{2} \backslash K_{1} ; x_{1}, b\right) .
$$

By Propn. 6.5, the law of $K_{1}$ is determined by Cardy's formula, and further $D_{2}^{\delta}(1) \rightsquigarrow D_{2}(1)$ which means that we can repeat the construction in the new admissible domain $D_{2}(1)$. Continuing in this way the result is proved.

\section{Conclusion}

Since the introduction of SLE in [94], a number of discrete interface models have been shown to have scaling limits which are versions of SLE. We have focused on a particular model throughout this article, so we conclude with a listing of other examples. The following results are proved:

- $\mathrm{SLE}_{2}$ is the scaling limit of the loop-erased random walk, and $\mathrm{SLE}_{8}$ is the scaling limit of the uniform spanning tree Peano path (Lawler, Schramm, Werner [77, 94]).

- $\mathrm{SLE}_{6}$ is the scaling limit of the percolation exploration path (Smirnov [105, 108], Camia and Newman [31]). The scaling limit of the full configuration is determined in [30].

The outer boundary of $\mathrm{SLE}_{6}$ is $\mathrm{SLE}_{8 / 3}$, which is also the outer boundary of a certain reflected Brownian motion [71]; for a discussion of the connection see $[79]$.

- $\mathrm{SLE}_{4}$ is the scaling limit of the path of the harmonic explorer and of the contour lines of the two-dimensional discrete Gaussian free field (GFF) with certain boundary conditions (Schramm and Sheffield [97, 98], extended to more general models by Miller [84, 85]). There is also a welldefined sense in which $\mathrm{SLE}_{4}$ is a contour line of the two-dimensional continuum GFF, again with certain boundary conditions [99]. In forthcoming work Miller and Sheffield [86] prove that the collection of all discrete GFF contours at certain heights converge to $\mathrm{CLE}_{4}$.

- $\mathrm{SLE}_{3}$ is the scaling limit of interfaces in the critical Ising spin model, and $\mathrm{SLE}_{16 / 3}$ is the scaling limit of interfaces in the corresponding FK cluster representation with parameters $q=2, p=1-e^{-2 \beta_{c}}[36,37,59,107,109$, $111]$.

The relationship between $\kappa$ and $16 / \kappa$ that is evident in this list is a manifestation of the Duplantier duality; see [39, 41, 119, 120].

The following results are conjectured; for more see $[92, \S 9]$ : 
- Chordal $\mathrm{SLE}_{8 / 3}$ is the scaling limit of the self-avoiding walk in a half-plane $\delta \Lambda \cap \overline{\mathbb{H}}$ started at 0 [71]. For recent progress see [43].

- $\mathrm{SLE}_{4}$ is the scaling limit of loops in the double dimer model. Recent support for this conjecture can be found in [63]. Results on the conformal invariance of dimers are in [60-62].

- The uniform measure on simple grid paths joining a boundary point to an interior point (joining two fixed boundary points) converges to radial (chordal) $\mathrm{SLE}_{8}[92, \S 9]$.

- $\mathrm{SLE}_{\kappa(q)}$ is the scaling limit of boundaries in the critical FK cluster model with parameters $q$ and $p=p(q)=\sqrt{q} /(1+\sqrt{q})$, where $q \in(0,4)$ and $\kappa(q)=4 \pi / \arccos (-\sqrt{q} / 2) \in(4,8)[92, \S 9]$. (The case $q=2$, corresponding to $\kappa=16 / 3$, has been solved by Smirnov.)

- $\mathrm{SLE}_{\kappa}$ is the scaling limit of a form of the $O(n)$ loop model for $8 / 3 \leq \kappa \leq 8$, $n=-2 \cos (4 \pi / \kappa)[56,102]$.

- Loops in the critical XOR-Ising model (the product of two independent critical Ising spin configurations), and more generally in the critical AshkinTeller model, converge to a form of $\mathrm{SLE}_{4}[55,118]$.

\section{References}

[1] Ahlfors, L. V. (1978). Complex analysis, Third ed. McGraw-Hill Book Co., New York. An introduction to the theory of analytic functions of one complex variable, International Series in Pure and Applied Mathematics. MR510197 (80c:30001)

[2] Ahlfors, L. V. (2010). Conformal invariants. AMS Chelsea Publishing, Providence, RI. Topics in geometric function theory, Reprint of the 1973 original, With a foreword by Peter Duren, F. W. Gehring and Brad Osgood. MR2730573

[3] Aizenman, M. (1997). On the number of incipient spanning clusters. Nuclear Phys. B 485, 3, 551-582. http://dx.doi.org/10.1016/ S0550-3213(96)00626-8. MR1431856 (98j:82030)

[4] Aizenman, M. (1998). Scaling limit for the incipient spanning clusters. In Mathematics of multiscale materials (Minneapolis, MN, 19951996). IMA Vol. Math. Appl., Vol. 99. Springer, New York, 1-24. MR1635999 (99g:82034)

[5] Aizenman, M. and Burchard, A. (1999). Hölder regularity and dimension bounds for random curves. Duke Math. J. 99, 3, 419-453. http://dx. doi.org/10.1215/S0012-7094-99-09914-3. MR1712629 (2000i:60012)

[6] Aizenman, M., Duplantier, B., And Aharony, A. (1999). Pathcrossing exponents and the external perimeter in 2D percolation. Phys. Rev. Lett. 83, 7 (Aug), 1359-1362.

[7] Aizenman, M., Kesten, H., And Newman, C. M. (1987). Uniqueness of the infinite cluster and continuity of connectivity functions for short and long range percolation. Comm. Math. Phys. 111, 4, 505-531. http://projecteuclid.org/getRecord?id=euclid.cmp/1104159720.

MR901151 (89b:82060) 
[8] Alon, N., Benjamini, I., And Stacey, A. (2004). Percolation on finite graphs and isoperimetric inequalities. Ann. Probab. 32, 3A, 1727-1745. http://dx.doi.org/10.1214/009117904000000414. MR2073175 (2005f:05149)

[9] Beffara, V. (2004). Hausdorff dimensions for $\mathrm{SLE}_{6}$. Ann. Probab. 32, 3B, 2606-2629. http://dx.doi.org/10.1214/009117904000000072. MR2078552 (2005k:60295)

[10] Beffara, V. (2007). Cardy's formula on the triangular lattice, the easy way. In Universality and renormalization. Fields Inst. Commun., Vol. 50. Amer. Math. Soc., Providence, RI, 39-45. MR2310300 (2008i:82030)

[11] Beffara, V. (2008a). The dimension of the SLE curves. Ann. Probab. 36, 4, 1421-1452. http://dx.doi.org/10.1214/07-AOP364. MR2435854 (2009e:60026)

[12] Beffara, V. (2008b). Is critical 2D percolation universal? In In and out of equilibrium. 2. Progr. Probab., Vol. 60. Birkhäuser, Basel, 31-58. http://dx.doi.org/10.1007/978-3-7643-8786-0_3. MR2477376 (2010h:60262)

[13] Beffara, V. And Duminil-Copin, H. (2011). Planar percolation with a glimpse of Schramm-Loewner evolution. ArXiv preprint.

[14] Benjamini, I. and Kozma, G. (2011). ZZ-actions and uniqueness of percolation. ArXiv preprint.

[15] Benjamini, I., Lyons, R., Peres, Y., and Schramm, O. (1999a). Critical percolation on any nonamenable group has no infinite clusters. Ann. Probab. 27, 3, 1347-1356. http://dx.doi.org/10.1214/aop/1022677450. MR1733151 (2000k:60197)

[16] Benjamini, I., Lyons, R., Peres, Y., and Schramm, O. (1999b). Group-invariant percolation on graphs. Geom. Funct. Anal. 9, 1, 29-66. http://dx.doi.org/10.1007/s000390050080. MR1675890 (99m:60149)

[17] Benjamini, I. And Schramm, O. (1998). Conformal invariance of Voronoi percolation. Comm. Math. Phys. 197, 1, 75-107. http://dx.doi.org/10.1007/s002200050443. MR1646475 (99i:60172)

[18] Billingsley, P. (1999). Convergence of probability measures, Second ed. Wiley Series in Probability and Statistics: Probability and Statistics. John Wiley \& Sons Inc., New York. A Wiley-Interscience Publication, http://dx.doi.org/10.1002/9780470316962. MR1700749 (2000e:60008)

[19] Binder, I., Chayes, L., And Lei, H. K. (2010a). On convergence to $\mathrm{SLE}_{6}$ I: conformal invariance for certain models of the bond-triangular type. J. Stat. Phys. 141, 2, 359-390. http://dx.doi.org/10.1007/ s10955-010-0052-3. MR2726646

[20] Binder, I., Chayes, L., And Lei, H. K. (2010b). On convergence to $\mathrm{SLE}_{6}$ II: discrete approximations and extraction of Cardy's formula for general domains. J. Stat. Phys. 141, 2, 391-408. http://dx.doi.org/10.1007/ s10955-010-0053-2. MR2726647

[21] Bollobás, B. (2001). Random graphs, Second ed. Cambridge Studies in Advanced Mathematics, Vol. 73. Cambridge University Press, Cambridge. MR1864966 (2002j:05132) 
[22] Bollobás, B. and Kohayakawa, Y. (1994). Percolation in high dimensions. European J. Combin. 15, 2, 113-125. http://dx.doi.org/10.1006/eujc. 1994.1014. MR1261058 (95c:60092)

[23] Bollobás, B. and Riordan, O. (2006). Percolation. Cambridge University Press, New York. MR2283880 (2008c:82037)

[24] Borgs, C., Chayes, J. T., Kesten, H., And Spencer, J. (2001). The birth of the infinite cluster: finite-size scaling in percolation. Comm. Math. Phys. 224, 1, 153-204. Dedicated to Joel L. Lebowitz, http://dx.doi.org/ 10.1007/s002200100521. MR1868996 (2002k:60199)

[25] Borgs, C., Chayes, J. T., van der Hofstad, R., Slade, G., AND Spencer, J. (2005a). Random subgraphs of finite graphs. I. The scaling window under the triangle condition. Random Structures Algorithms 27, 2, 137-184. http://dx.doi.org/10.1002/rsa.20051. MR2155704 (2006e:05156)

[26] Borgs, C., Chayes, J. T., van der Hofstad, R., Slade, G., And Spencer, J. (2005b). Random subgraphs of finite graphs. II. The lace expansion and the triangle condition. Ann. Probab. 33, 5, 1886-1944. http://dx.doi.org/10.1214/009117905000000260. MR2165583 (2006j:60009)

[27] Borgs, C., Chayes, J. T., van der Hofstad, R., Slade, G., And Spencer, J. (2006). Random subgraphs of finite graphs. III. The phase transition for the n-cube. Combinatorica 26, 4, 395-410. http://dx.doi.org/10.1007/s00493-006-0022-1. MR2260845 (2007k:05194)

[28] Broadbent, S. R. and Hammersley, J. M. (1957). Percolation processes. I. Crystals and mazes. Proc. Cambridge Philos. Soc. 53, 629-641. MR0091567 (19,989e)

[29] Burton, R. M. and Keane, M. (1989). Density and uniqueness in percolation. Comm. Math. Phys. 121, 3, 501-505. http://projecteuclid.org/ getRecord?id=euclid.cmp/1104178143. MR990777 (90g:60090)

[30] Camia, F. And Newman, C. M. (2006). Two-dimensional critical percolation: the full scaling limit. Comm. Math. Phys. 268, 1, 1-38. http://dx.doi.org/10.1007/s00220-006-0086-1. MR2249794 (2007m:82032)

[31] Camia, F. and Newman, C. M. (2007). Critical percolation exploration path and $\mathrm{SLE}_{6}$ : a proof of convergence. Probab. Theory Related Fields 139, 3-4, 473-519. http://dx.doi.org/10.1007/s00440-006-0049-7. MR2322705 (2008k:82040)

[32] Carathéodory, C. (1913). Über die Begrenzung einfach zusammenhängender Gebiete. Math. Ann. 73, 3, 323-370. http://dx.doi.org/ 10.1007/BF01456699. MR1511737

[33] CARdy, J. (2001). Conformal invariance and percolation. ArXiv preprint.

[34] Cardy, J. L. (1992). Critical percolation in finite geometries. J. Phys. A 25, 4, L201-L206. http://stacks.iop.org/0305-4470/25/L201. MR1151081 (92m:82048)

[35] Chayes, L. and Lei, H. K. (2007). Cardy's formula for certain models of the bond-triangular type. Rev. Math. Phys. 19, 5, 511-565. http://dx.doi.org/10.1142/S0129055X0700305X. MR2337476 (2008j:82019) 
[36] Chelkak, D. and Smirnov, S. (2009). Universality in the 2D Ising model and conformal invariance of fermionic observables. ArXiv preprint.

[37] Chelkak, D. And Smirnov, S. (2010). Conformal invariance of the 2D Ising model at criticality. In preparation.

[38] Ding, J., Kim, J. H., Lubetzky, E., And Peres, Y. (2011). Anatomy of a young giant component in the random graph. Random Structures Algorithms 39, 2, 139-178. http://dx.doi.org/10.1002/rsa.20342.

[39] Dubédat, J. (2005). $\operatorname{SLE}(\kappa, \rho)$ martingales and duality. Ann. Probab. 33, 1, 223-243. http://dx.doi.org/10.1214/009117904000000793. MR2118865 (2005j:60180)

[40] Dubédat, J. (2006). Excursion decompositions for SLE and Watts' crossing formula. Probab. Theory Related Fields 134, 3, 453-488. http://dx.doi.org/10.1007/s00440-005-0446-3. MR2226888 (2007d:60019)

[41] Dubédat, J. (2009). Duality of Schramm-Loewner evolutions. Ann. Sci. Éc. Norm. Supér. (4) 42, 5, 697-724. MR2571956 (2011g:60151)

[42] Dudley, R. M. (1968). Distances of probability measures and random variables. Ann. Math. Statist 39, 1563-1572. MR0230338 (37 \#5900)

[43] Duminil-Copin, H. And Smirnov, S. (2010). The connective constant of the honeycomb lattice equals $\sqrt{2+\sqrt{2}}$. ArXiv preprint.

[44] Duminil-Copin, H. And Smirnov, S. (2011). Conformal invariance of lattice models. ArXiv preprint.

[45] Epstein, D. B. A. (1981). Prime ends. Proc. London Math. Soc. (3) 42, 3, 385-414. http://dx.doi.org/10.1112/plms/s3-42.3.385. MR614728 (83c:30025)

[46] Fortuin, C. M., Kasteleyn, P. W., and Ginibre, J. (1971). Correlation inequalities on some partially ordered sets. Comm. Math. Phys. 22, 89-103. MR0309498 (46 \#8607)

[47] Grimmett, G. (1999). Percolation, Second ed. Grundlehren der Mathematischen Wissenschaften, Vol. 321. Springer-Verlag, Berlin. MR1707339 (2001a:60114)

[48] Grimmett, G. (2010). Probability on graphs. Institute of Mathematical Statistics Textbooks, Vol. 1. Cambridge University Press, Cambridge. Random processes on graphs and lattices. MR2723356

[49] Grimmett, G. R. and Stacey, A. M. (1998). Critical probabilities for site and bond percolation models. Ann. Probab. 26, 4, 1788-1812. http://dx.doi.org/10.1214/aop/1022855883. MR1675079 (2000d:60160)

[50] Hara, T. and Slade, G. (1990). Mean-field critical behaviour for percolation in high dimensions. Comm. Math. Phys. 128, 2, 333-391. http://projecteuclid.org/getRecord?id=euclid.cmp/1104180434. MR1043524 (91a:82037)

[51] Hara, T. and Slade, G. (1994). Mean-field behaviour and the lace expansion. In Probability and phase transition (Cambridge, 1993). NATO Adv. Sci. Inst. Ser. C Math. Phys. Sci., Vol. 420. Kluwer Acad. Publ., Dordrecht, 87-122. MR1283177 (95d:82033) 
[52] Hara, T. And Slade, G. (1995). The self-avoiding-walk and percolation critical points in high dimensions. Combin. Probab. Comput. 4, 3, 197-215. http://dx.doi.org/10.1017/S0963548300001607. MR1356575 (96i:82081)

[53] HARris, T. E. (1960). A lower bound for the critical probability in a certain percolation process. Proc. Cambridge Philos. Soc. 56, 13-20. MR0115221 (22 \#6023)

[54] Hongler, C. And Smirnov, S. (2010). The energy density in the planar Ising model. ArXiv preprint.

[55] IKhlef, Y. And Rajabpour, M. A. (2011). Discrete holomorphic parafermions in the Ashkin-Teller model and SLE. J. Phys. A 44, 4, 042001, 11. http://dx.doi.org/10.1088/1751-8113/44/4/042001. MR2754707

[56] Kager, W. And Nienhuis, B. (2004). A guide to stochastic Löwner evolution and its applications. J. Statist. Phys. 115, 5-6, 1149-1229. http://dx.doi.org/10.1023/B:JOSS.0000028058.87266.be. MR2065722 (2005f:82037)

[57] Kakutani, S. (1944). Two-dimensional Brownian motion and harmonic functions. Proc. Imp. Acad. Tokyo 20, 706-714. MR0014647 (7,315b)

[58] Karatzas, I. And Shreve, S. E. (1991). Brownian motion and stochastic calculus, Second ed. Graduate Texts in Mathematics, Vol. 113. Springer-Verlag, New York. MR1121940 (92h:60127)

[59] Kemppainen, A. and Smirnov, S. (2011). Conformal invariance in random cluster models. III. Full scaling limit. In preparation.

[60] Kenyon, R. (2000). Conformal invariance of domino tiling. Ann. Probab. 28, 2, 759-795. http://dx.doi.org/10.1214/aop/1019160260. MR1782431 (2002e:52022)

[61] Kenyon, R. (2001). Dominos and the Gaussian free field. Ann. Probab. 29, 3, 1128-1137. http://dx.doi.org/10.1214/aop/1015345599. MR1872739 (2002k:82039)

[62] Kenyon, R. (2011). Conformal invariance of loops in the double-dimer model. ArXiv preprint.

[63] Kenyon, R. W. and Wilson, D. B. (2011). Boundary partitions in trees and dimers. Trans. Amer. Math. Soc. 363, 3, 1325-1364. http://dx. doi.org/10.1090/S0002-9947-2010-04964-5. MR2737268 (2011i:60023)

[64] Kesten, H. (1980). The critical probability of bond percolation on the square lattice equals $\frac{1}{2}$. Comm. Math. Phys. 74, 1, 41-59. http://projecteuclid.org/getRecord?id=euclid.cmp/1103907931. MR575895 (82c:60179)

[65] Kesten, H. (1982). Percolation theory for mathematicians. Progress in Probability and Statistics, Vol. 2. Birkhäuser Boston, Mass. MR692943 (84i:60145)

[66] Kesten, H. (1990). Asymptotics in high dimensions for percolation. In Disorder in physical systems. Oxford Sci. Publ. Oxford Univ. Press, New York, 219-240. MR1064563 (91k:60114)

[67] Kesten, H., Sidoravicius, V., And Zhang, Y. (1998). Almost all words are seen in critical site percolation on the triangular lattice. Electron. J. Probab. 3, no. 10, 75 pp. (electronic). http://www.math.washington. 
edu/ ejpecp/EjpVol3/paper10.abs.html. MR1637089 (99j:60155)

[68] Lalley, S., Lawler, G., and Narayanan, H. (2009). Geometric interpretation of half-plane capacity. Electron. Commun. Probab. 14, 566-571. MR2576752 (2011b:60332)

[69] Langlands, R., Pouliot, P., And Saint-Aubin, Y. (1994). Conformal invariance in two-dimensional percolation. Bull. Amer. Math. Soc. (N.S.) 30, 1, 1-61. http://dx.doi.org/10.1090/S0273-0979-1994-00456-2. MR1230963 (94e:82056)

[70] Lawler, G. (2009). Schramm-Loewner evolution (SLE). In Statistical mechanics. IAS/Park City Math. Ser., Vol. 16. Amer. Math. Soc., Providence, RI, 231-295. MR2523461 (2011d:60244)

[71] Lawler, G., Schramm, O., And Werner, W. (2003). Conformal restriction: the chordal case. J. Amer. Math. Soc. 16, 4, 917-955 (electronic). http://dx.doi.org/10.1090/S0894-0347-03-00430-2. MR1992830 (2004g:60130)

[72] LAWLER, G. F. (2005). Conformally invariant processes in the plane. Mathematical Surveys and Monographs, Vol. 114. American Mathematical Society, Providence, RI. MR2129588 (2006i:60003)

[73] Lawler, G. F., Schramm, O., And Werner, W. (2001a). Values of Brownian intersection exponents. I. Half-plane exponents. Acta Math. 187, 2, 237-273. http://dx.doi.org/10.1007/BF02392618. MR1879850 (2002m:60159a)

[74] Lawler, G. F., Schramm, O., And Werner, W. (2001b). Values of Brownian intersection exponents. II. Plane exponents. Acta Math. 187, 2, 275-308. http://dx.doi.org/10.1007/BF02392619. MR1879851 (2002m:60159b)

[75] Lawler, G. F., Schramm, O., and Werner, W. (2002a). One-arm exponent for critical 2D percolation. Electron. J. Probab. 7, no. 2, 13 pp. (electronic). http://www.math.washington.edu/ ejpecp/EjpVol7/paper2. abs.html. MR1887622 (2002k:60204)

[76] Lawler, G. F., Schramm, O., And Werner, W. (2002b). Values of Brownian intersection exponents. III. Two-sided exponents. Ann. Inst. H. Poincaré Probab. Statist. 38, 1, 109-123. http://dx.doi.org/10.1016/ S0246-0203(01)01089-5. MR1899232 (2003d:60163)

[77] Lawler, G. F., Schramm, O., And Werner, W. (2004). Conformal invariance of planar loop-erased random walks and uniform spanning trees. Ann. Probab. 32, 1B, 939-995. http://dx.doi.org/10.1214/aop/1079021469. MR2044671 (2005f:82043)

[78] LAWler, G. F. AND ShefField, S. (2009). The natural parametrization for the Schramm-Loewner evolution. ArXiv preprint.

[79] LAWler, G. F. AND Werner, W. (2000). Universality for conformally invariant intersection exponents. J. Eur. Math. Soc. (JEMS) 2, 4, 291328. http://dx.doi.org/10.1007/s100970000024. MR1796962 (2002g:60123)

[80] LÉvy, P. (1992). Processus stochastiques et mouvement brownien. Les Grands Classiques Gauthier-Villars. Éditions Jacques Gabay, Sceaux. Followed by a note by M. Loève, Reprint of the second (1965) edition. MR1188411 (93i:60003) 
[81] Lieb, E. H. And Loss, M. (2001). Analysis, Second ed. Graduate Studies in Mathematics, Vol. 14. American Mathematical Society, Providence, RI. MR1817225 (2001i:00001)

[82] Marshall, D. E. And Rohde, S. (2005). The Loewner differential equation and slit mappings. J. Amer. Math. Soc. 18, 4, 763-778 (electronic). http://dx.doi.org/10.1090/S0894-0347-05-00492-3. MR2163382 (2006d:30022)

[83] Mendelson, D., Nachmias, A., Sheffield, S., and Watson, S. (2011). Convergence rate in Cardy's formula. In preparation.

[84] Miller, J. (2010a). Fluctuations for the Ginzburg-Landau $\nabla \phi$ interface model on a bounded domain. ArXiv preprint.

[85] Miller, J. (2010b). Universality for SLE(4). ArXiv preprint.

[86] Miller, J. And Sheffield, S. (2011). CLE(4) and the Gaussian free field. In preparation.

[87] Mörters, P. And Peres, Y. (2010). Brownian motion. Cambridge Series in Statistical and Probabilistic Mathematics. Cambridge University Press, Cambridge. With an appendix by Oded Schramm and Wendelin Werner. MR2604525

[88] Munkres, J. R. (2000). Topology, Second ed. Prentice Hall, Upper Saddle River, NJ.

[89] Nachmias, A. (2009). Mean-field conditions for percolation on finite graphs. Geom. Funct. Anal. 19, 4, 1171-1194. http://dx.doi.org/10.1007/ s00039-009-0032-4. MR2570320 (2011e:60228)

[90] Pommerenke, C. (1992). Boundary behaviour of conformal maps. Grundlehren der Mathematischen Wissenschaften, Vol. 299. SpringerVerlag, Berlin. MR1217706 (95b:30008)

[91] Reimer, D. (2000). Proof of the van den Berg-Kesten conjecture. Combin. Probab. Comput. 9, 1, 27-32. http://dx.doi.org/10.1017/S0963548399004113. MR1751301 (2001g:60017)

[92] Rohde, S. and Schramm, O. (2005). Basic properties of SLE. Ann. of Math. (2) 161, 2, 883-924. http://dx.doi.org/10.4007/annals.2005.161.883. MR2153402 (2006f:60093)

[93] Russo, L. (1978). A note on percolation. Z. Wahrscheinlichkeitstheorie und Verw. Gebiete 43, 1, 39-48. MR0488383 (58 \#7931)

[94] Schramm, O. (2000). Scaling limits of loop-erased random walks and uniform spanning trees. Israel J. Math. 118, 221-288. http://dx.doi.org/10. 1007/BF02803524. MR1776084 (2001m:60227)

[95] Schramm, O. (2001). A percolation formula. Electron. Comm. Probab. 6, 115-120 (electronic). MR1871700 (2002h:60227)

[96] Schramm, O. (2007). Conformally invariant scaling limits: an overview and a collection of problems. In International Congress of Mathematicians. Vol. I. Eur. Math. Soc., Zürich, 513-543. http://dx.doi.org/10.4171/022-1/20. MR2334202 (2008j:60237)

[97] Schramm, O. and Sheffield, S. (2005). Harmonic explorer and its convergence to SLE 4 . Ann. Probab. 33, 6, 2127-2148. http://dx.doi.org/10. 1214/009117905000000477. MR2184093 (2006i:60013) 
[98] Schramm, O. and Sheffield, S. (2009). Contour lines of the twodimensional discrete Gaussian free field. Acta Math. 202, 1, 21-137. http://dx.doi.org/10.1007/s11511-009-0034-y. MR2486487 (2010f:60238)

[99] Schramm, O. And Sheffield, S. (2010). A contour line of the continuum Gaussian free field. ArXiv preprint.

[100] Schramm, O. And Wilson, D. B. (2005). SLE coordinate changes. New York J. Math. 11, 659-669 (electronic). http://nyjm.albany.edu:8000/j/ 2005/11_659.html. MR2188260 (2007e:82019)

[101] Seymour, P. D. And Welsh, D. J. A. (1978). Percolation probabilities on the square lattice. Ann. Discrete Math. 3, 227-245. Advances in graph theory (Cambridge Combinatorial Conf., Trinity College, Cambridge, 1977). MR0494572 (58 \#13410)

[102] Sheffield, S. (2009). Exploration trees and conformal loop ensembles. Duke Math. J. 147, 1, 79-129. http://dx.doi.org/10.1215/00127094-2009-007. MR2494457 (2010g:60184)

[103] Sheffield, S. And Werner, W. (2010). Conformal loop ensembles: The Markovian characterization and the loop-soup construction. To appear, Ann. of Math.

[104] Sheffield, S. And Wilson, D. B. (2010). Schramm's proof of Watts' formula. ArXiv preprint.

[105] Smirnov, S. (2001). Critical percolation in the plane: conformal invariance, Cardy's formula, scaling limits. C. R. Acad. Sci. Paris Sér. I Math. 333, 3, 239-244. http://dx.doi.org/10.1016/S0764-4442(01)01991-7. MR1851632 (2002f:60193)

[106] Smirnov, S. (2005). Critical percolation and conformal invariance. In XIVth International Congress on Mathematical Physics. World Sci. Publ., Hackensack, NJ, 99-112. MR2227824 (2007h:82030)

[107] Smirnov, S. (2006). Towards conformal invariance of 2D lattice models. In International Congress of Mathematicians. Vol. II. Eur. Math. Soc., Zürich, 1421-1451. MR2275653 (2008g:82026)

[108] Smirnov, S. (2009). Critical percolation in the plane. ArXiv preprint.

[109] Smirnov, S. (2010a). Conformal invariance in random cluster models. I. Holomorphic fermions in the Ising model. Ann. of Math. (2) 172, 2, 1435-1467. http://dx.doi.org/10.4007/annals.2010.172.1441. MR2680496

[110] Smirnov, S. (2010b). Discrete complex analysis and probability. ArXiv preprint.

[111] Smirnov, S. (2011). Conformal invariance in random cluster models. II. Scaling limit of the interface. In preparation.

[112] Stauffer, D. And Aharony, A. (1992). Introduction to percolation theory, 2nd ed. Taylor \& Francis, London.

[113] Stein, E. M. and Shakarchi, R. (2003a). Complex analysis. Princeton Lectures in Analysis, II. Princeton University Press, Princeton, NJ. MR1976398 (2004d:30002)

[114] Stein, E. M. and Shakarchi, R. (2003b). Fourier analysis. Princeton Lectures in Analysis, Vol. 1. Princeton University Press, Princeton, NJ. An introduction. MR1970295 (2004a:42001) 
[115] van den Berg, J. And Kesten, H. (1985). Inequalities with applications to percolation and reliability. J. Appl. Probab. 22, 3, 556-569. MR799280 (87b:60027)

[116] Werner, W. (2004). Random planar curves and Schramm-Loewner evolutions. In Lectures on probability theory and statistics. Lecture Notes in Math., Vol. 1840. Springer, Berlin, 107-195. MR2079672 (2005m:60020)

[117] Werner, W. (2009). Lectures on two-dimensional critical percolation. In Statistical mechanics. IAS/Park City Math. Ser., Vol. 16. Amer. Math. Soc., Providence, RI, 297-360. MR2523462 (2011e:82042)

[118] Wilson, D. B. (2011). XOR-Ising loops and the Gaussian free field. ArXiv preprint.

[119] Zhan, D. (2008). Duality of chordal SLE. Invent. Math. 174, 2, 309-353. http://dx.doi.org/10.1007/s00222-008-0132-z. MR2439609 (2010f:60239)

[120] Zhan, D. (2010). Duality of chordal SLE, II. Ann. Inst. Henri Poincaré Probab. Stat. 46, 3, 740-759. http://dx.doi.org/10.1214/09-AIHP340. MR2682265 